\title{
ARTÍCULO/ARTICLE
}

\section{Memorias de la V Reunión Ecuatoriana de Ornitología}

Leonardo Ordóñez-Delgado ${ }^{1,2}$, Juan F. Freile ${ }^{2,3^{*}}$, Esteban A. Guevara ${ }^{2,4}$, Diego F. Cisneros-Heredia ${ }^{2,4,5}$ y Tatiana Santander ${ }^{2,4}$

${ }^{1}$ Departamento de Ciencias Biológicas, Universidad Técnica Particular de Loja, San Cayetano Alto, Calle París, Loja, Ecuador.

2 Red Aves Ecuador.

${ }^{3}$ Comité Ecuatoriano de Registros Ornitológicos, Pasaje El Moro E4-216 y Norberto Salazar, Tumbaco, Ecuador.

${ }^{4}$ Aves y Conservación - BirdLife en Ecuador, Pasaje Joaquín Tinajero E3-05 y Jorge Drom, Quito, Ecuador.

${ }^{5}$ Universidad San Francisco de Quito, Colegio de Ciencias Biológicas y Ambientales, Laboratorio de Zoología Terrestre, Calle Diego de Robles y Vía Interoceánica, campus Cumbayá, Edif. Darwin, of. DW-010A, Casilla Postal 17-1200-841, Quito, Ecuador.

\author{
Editado por/Edited by: Juan Freile, Esteban Guevara y Diego Cisneros-Heredia \\ Recibido/Received: 25/08/2016. Aceptado/Accepted: 03/03/2017. \\ Publicado en línea/Published online: 08/02/2018
}

DOI: http://dx.doi.org/10.18272/reo.v0i2.843

\section{Abstracts of the V Ecuadorian Ornithology Meeting}

\section{Resumen}

Presentamos las memorias de la V Reunión Ecuatoriana de Ornitología (V REO), desarrollada en Zamora, en 11-14 Agosto 2016. La V REO contó con cinco charlas magistrales, un simposio sobre aves, café y cacao, 46 presentaciones orales y pósters, conversatorios sobre el Festival Mundial de las Aves, conteos navideños y Global Big Day, un taller de escritura científica, un taller de ilustración de aves en acuarela y una discusión abierta sobre la conformación de la Red Aves Ecuador. Contó con casi 150 participantes, entre expositores y asistentes.

Palabras clave: Investigación, difusión, ornitología, conservación, Ecuador.

\begin{abstract}
We present the abstracts of the V Ecuadorian Ornithology Meeting (V REO), held in Zamora, on 11-14 August 2016. The V REO included five plenary talks, a symposium on birds, coffee and cacao, 46 oral and poster presentations, round tables about the World Birds Festival, the Christmas bird counts and Global Big Days, a workshop on scientific writing, a workshop on bird illustration in watercolor, and an open discussion session about the creation of Red Aves Ecuador. Nearly 150 contributors and assistants participated in the V REO.
\end{abstract}

Keywords: Conservation, divulgation, Ecuador, ornithology, research. 


\section{CONVOCA}

Red Aves Ecuador

\section{Organizadores}

Gobierno Autónomo Descentralizado Provincial de Zamora Chinchipe

Universidad Técnica Particular de Loja

Naturaleza y Cultura Internacional

Copalinga Ecolodge

Cámara de Turismo de Zamora Chinchipe

Gobierno Autónomo Descentralizado Municipal de Zamora

\section{Comité Organizador Local}

Paúl Palacios Toledo (Gobierno Provincial de Zamora Chinchipe)

Génesis Romero (Gobierno Provincial de Zamora Chinchipe)

Leonardo Ordóñez-Delgado (Universidad Técnica Particular de Loja)

Trotsky Riera (Naturaleza y Cultura Internacional).

Catherine Vits (Copalinga Ecolodge)

Wellington Valdivieso (Cámara de Turismo de Zamora Chinchipe)

Hilter Sarango (Gobierno Municipal de Zamora)

\section{Comité Organizador Nacional:}

Juan F. Freile (Comité Ecuatoriano de Registros Ornitológicos)

Tatiana Santander (Fundación Aves y Conservación)

Esteban Guevara (Fundación Aves y Conservación)

José María Loaiza (Red Aves Ecuador)

Xavier Amigo (Ecuador Experience)

Diego F. Cisneros-Heredia (Universidad San Francisco de Quito \& Aves y Conservación)

\section{Auspiciantes}

Ecuanativa Cia. Ltda.

Cámara de la Cultura del Ecuador

Aves y Conservación - BirdLife Ecuador

Nature Experience

Ecología Terrestre

Tandayapa Bird Lodge

Ecuador Terra Incognita

Tropical Birding

Fundación de Conservación Jocotoco

Green Team - US Embassy 


\title{
CHARLAS MAGISTRALES
}

\section{AÑOS DE AVES EN ECUADOR: UN VIAJE DE EXPLORACIÓN Y DESCUBRIMIENTO}

\author{
Paul J. Greenfield \\ Mindo Cloudforest Foundation, Camino Milpe-Pachijal s/n \\ y km 91 Vía Calacalí - La Independencia, Ecuador. \\ E-mail: greenfield.p@gmail.com
}

Describo de manera breve y personal el "contraste" entre el estado actual del pajareo y el Ecuador que conocí cuando llegué a Guayaquil a principios de la década de 1970, cuando no existía la infraestructura aviturística que disfrutamos hoy, y cuando encontrar e identificar las especies de aves -incluso algunas que ahora consideramos "fáciles"- era todo un reto. No existían libros, guías de campo, CD de audio, Apps, ni páginas web dedicadas a las aves como tenemos actualmente. La calidad y disponibilidad de los equipos de campo (binoculares, telescopios) era muy limitado. No existían las computadoras o cámaras digitales. Ecuador y sus aves eran prácticamente desconocidos a nivel mundial. Reflexiono sobre algunas anécdotas "del camino". Menciono sitios de observación "descubiertos" en aquel entonces -algunos, ahora clásicos y otros lamentablemente olvidados- y algunas especies descubiertas. Señalo algunos amigos, compañeros, profesionales y socios que formaban parte del increíble viaje por este país mágico, aunque siempre amenazado por políticas contrarias, la deforestación, incendios forestales y la cacería de aquel tiempo. Presento los años de formación desde "cero": las décadas de 1980 y 1990, época de exploración y expediciones (tanto informales como organizadas) alrededor del territorio nacional; el reto y trabajo de elaborar el libro de Birds of Ecuador junto a Robert Ridgely y las instituciones que nos apoyaron. La verdad es que yo no tenía idea del significado que llegaría a tener este emprendimiento, ni cómo concluiría, pero creo se logró aportar a los conocimientos sobre la avifauna ecuatoriana con importantes descubrimientos para el país y para la ciencia.

\section{Palabras clave}

Aviturismo, historia, ornitología, pajareo, Ecuador.

\section{AVES Y CAFÉ: DESDE CONTROL DE PLAGAS HASTA DIVERSIFICACIÓN ECONÓMICA}

\section{Ernesto M. Carman}

Get Your Birds!, 1-7100 Paraíso, 30201, Costa Rica.

E-mail: emcarman@gmail.com

El café se ha convertido en uno de los productos más comercializados del mundo. No obstante, su producción se ha tornado más difícil en ciertas regiones del mundo. Cambio climático, plagas y precios bajos han hecho que el cultivo de café, especialmente a pequeña escala, haya disminuido enormemente. Esto ha llevado a la necesidad de diversificar la actividad económica de las fincas utilizando distintos métodos, como la búsqueda de nichos especializados en el mercado. Un ejemplo es el café orgánico o mediante métodos de cultivo o procesamiento alternativos. Involucrar a las aves en el esquema económico del café puede traer beneficios por diversos frentes: 1) control de plagas, 2) atracción de avituristas y 3) herramienta de mercadeo.

\section{Palabras clave}

Aves, café, control de plagas, diversificación económica. 


\section{EL COMPORTAMIENTO Y PAPEL ECOLÓGICO DEL PÁJARO TORO Cephalopterus penduliger}

\section{Jordan Karubian}

Fundación para la Conservación de los Andes Tropicales (FCAT), Mariano Hurtado N 50-89 y Vicente Heredia, Quito, Ecuador.

Department of Ecology \& Evolutionary Biology, Tulane University, 400 Boggs Building, New Orleans, LA, 70118, Estados Unidos. E-mail: jk@tulane.edu

El Pájaro Paraguas Longuipéndulo Cephalopterus penduliger es conocido en comunidades rurales en la provincia de Esmeraldas como "pájaro toro" por su canto. Junto con Jorge Olivo, Domingo Cabrera y otros campesinos de la Reserva Ecológica Mache Chindul he estado estudiando el comportamiento de esta especie desde 2003 hasta el presente. Esta charla pretende resumir aspectos relevantes de su comportamiento, papel ecológico y conservación. El pájaro paraguas forma leks en los que varios machos se congregan para hacer despliegues reproductivos. Identificamos dos estrategias de lek distintas en los machos: 1) defender un territorio fijo dentro de un solo lek; 2) moverse entre leks sin mantener un territorio. Los machos "flotantes" mantuvieron un rango de movimientos mucho mayor que los machos territoriales (aproximadamente 500 ha versus 50 ha). En cambio, las hembras mantuvieron territorios individuales de aproximadamente 50 ha cada una. Como un ave frugívora grande, el pájaro toro es uno de los dispersores de semillas más importantes para árboles con semillas grandes como palmas y aguacates. Machos territoriales y hembras mueven semillas a distancias similares. Las hembras dispersan las semillas en una manera equitativa por todo su territorio, pero los machos territoriales producen concentraciones muy altas de semillas en los leks. Estas concentraciones pueden ser muy diversas, y no hemos notado que se reduzca la sobrevivencia de las plántulas a pesar de la alta densidad de individuos. Los machos flotantes, en cambio, pueden dispersar semillas a largas distancias y probablemente son responsables de casos en los cuales las semillas de un árbol madre han llegado a dos o más leks distintos. Muestreos en fragmentos aislados de bosque sugieren que Cephalopterus penduliger es sensible a disturbios humanos y que los árboles que dispersan pueden sufrir consecuencias negativas para la diversidad genética en la ausencia de este dispersor. El pájaro paraguas es un ave importante para la regeneración y mantenimiento de los bosques y es una fuente de orgullo para los residentes locales que lo conservan.

\section{Palabras clave}

Conservación, dispersión de semillas, fragmentación, lek, Cephalopterus penduliger, Reserva Ecológica

Mache Chindul.

\section{HISTORIA DE LA ORNITOLOGÍA PERUANA: ¿QUÉ HA SUCEDIDO EN LAS ÚLTIMAS DOS DÉCADAS?}

\section{Fernando Angulo-Pratolongo}

Centro de Ornitología y Biodiversidad, CORBIDI

Calle Santa Rita 105 Of. 2. Urb. Huertos de San Antonio Monterrico,

Surco, Lima, Perú.

E-mail: chamaepetes@gmail.com

En los últimos 20 años, la forma en que ha cambiado la ornitología y la afición por la observación de aves en Perú es asombrosa. Hemos pasado de ser un país con pocos aficionados a las aves, a ser el país que ganó la competencia mundial de aves registradas en un solo día (Global Big Day) en 2015 y 2016. Hemos pasado de un país en el que faltaban ornitólogos peruanos, a uno en que tenemos muchos y muy destacados. Cómo sucedió esto es algo que trataré de explicar, mostrando algunos avances en años recientes. Parte de este progreso se explica por la posibilidad de acceder a mayor tecnología (binoculares, cámaras de fotos, reproductores mp3), y por tener las guías de aves de Perú (publicada en inglés en 2007 y en español en 2010). Esto ha hecho que el acercamiento a las aves sea más amigable y fácil versus tiempos pasados, cuando era 
necesario lidiar con varias limitaciones. Otro factor que ha contribuido es el cambio en los canales de comunicación. Antes de 1995, la comunicación era a través de revistas, si es que se daba. Con la aparición y masificación del Internet y correo electrónico, la comunicación mejoró y se convirtió en fluida. Desde principios de la década de 2010, la comunicación masiva giró hacia el Facebook. Destaca el grupo "Aves del Perú", que agrupa a casi 7300 personas. Asimismo, otra forma de intercambio de información, de corte más científico -y también de relaciones humanas- han sido los congresos de ornitología. El primero se dio en Lima, en 1995. En 2011 se realizó en Cusco el VIII Congreso Nacional de Ornitología, en paralelo con el IX Congreso de Ornitología Neotropical. En este se batió el récord de asistencia (1200 asistentes). En mayo de 2016 fue el décimo congreso en Chachapoyas. Estas diez reuniones han servido para estrechar y fortalecer los lazos entre los interesados en las aves en Perú, así como para mostrar los avances en el conocimiento. En los últimos años, se ha combinado el evento científico puro con ferias, donde se exponen y venden productos relacionados al pajareo.

Con respecto a la producción científica sobre aves del Perú, hace dos décadas ésta era básicamente hecha por extranjeros, quienes visitaban temporalmente el país, y publicaban en inglés en revistas internacionales. Los peruanos no publicaban. Poco a poco la producción aumentó tanto en número de artículos escritos por peruanos como en el número de revistas para publicar. En 2009 nació el Boletín de la Unión de Ornitólogos del Perú (Boletín UNOP), que publica información sobre aves peruanas. Desde esa fecha hasta hoy se han editado 22 números, 105 artículos, muchos de ellos escritos por académicos, pero también por estudiantes, guardaparques, guías de turismo y montaña, y personas naturales, con datos valiosos sobre las aves del Perú. El Boletín UNOP se ha convertido en el vehículo de intercambio científico sobre aves del Perú. Al mismo tiempo, es un sitio de entrenamiento para que jóvenes profesionales pierdan el miedo a publicar en revistas científicas internacionales. Para muestra un botón: a la fecha, en el Boletín UNOP se han reportado 19 especies nuevas para Perú. Otra herramienta que ha revolucionado desde el 2008 la información sobre la distribución peruanas es eBird. Esta herramienta en línea, permite tener una mejor idea de la distribución de las aves en el país, ya que no solo se cuenta ahora con los registros hechos por científicos, sino que se incorporan los registros de observadores de aves y ciudadanos en general al conocimiento de las aves. La utilidad de esta herramienta está en crecimiento, y permite ahora subir fotos y cantos. Por ello, hace que cada observación cuente para planificar investigación, turismo y conservación.

Una de las limitantes que hemos tenido en Perú para el desarrollo de la ornitología y la observación de aves ha sido el acceso a la información científica, técnica y especializada. Mucha de esta información se ha publicado en revistas científicas y libros en inglés, en el extranjero (o simplemente, no se ha publicado). En el primer caso, era bastante difícil obtener la información. Con la aparición y uso masivo del correo electrónico y los documentos en PDF, se empezó a acceder a esta información de forma más regular. Sin embargo, desde la década de 1960, Manuel Plenge, un aficionado a las aves, ha venido recopilando la información referente a las aves del Perú: libros, artículos, reportes, tesis, revistas, en formato físico, y en los últimos años, en digital. Plenge mantiene al día la lista de las aves del Perú, la cual a partir del año 2009 se publica anualmente en la web del Boletín UNOP. Desde mayo de 2011 también se publica la Bibliografía de las aves del Perú, que se puede descargar gratis. A inicios de 2013, se publicó la lista de especies y subespecies, y en 2014 la lista de referencias bibliográficas. Estos documentos se actualizan anualmente y han ayudado a tener la información fresca y disponible.

En resumen, esta combinación de factores (y seguramente algunos más) han ayudado mucho al desarrollo de la ornitología, y han potenciado la investigación, el conocimiento y la conservación de las aves peruanas. Una década atrás, era impensable ver a un grupo de peruanos viajando al campo únicamente con el propósito de ver aves, y ahora ya no lo es.

\section{Palabras clave}

Aviturismo, historia, ornitología, Perú. 


\section{EL CANTO Y EL COLOR DE LAS AVES: EVOLUCIÓN DE LAS ESTRATEGIAS DE COMUNICACIÓN MULTIMODAL}

\section{Óscar Laverde}

Departamento de Biología, Universidad Nacional de Colombia, Bogotá, Colombia.

E-mail: oharaco@gmail.com

Los colores llamativos y los cantos elaborados de las aves han llamado la atención de investigadores, naturalistas, músicos, indígenas y campesinos. Dichas características están determinadas por las estrategias de comunicación que las aves han desarrollado para interactuar con individuos de su propia especie o de otras especies. El estudio de la comunicación en aves tradicionalmente se ha enfocado en entender la estructura de las señales acústicas y las características morfológicas del plumaje de forma independiente. Sin embargo, la comunicación es multimodal pues involucra la interacción de más de un canal de comunicación. Por otro lado, las características físicas del hábitat en que habitan las aves determinan los tipos de señales más óptimas para la trasmisión de la información. Parte de mi investigación involucra el estudio de las señales visuales y acústicas en la comunicación entre las aves y la relación que existe entre estas señales. Una explicación clásica propone una posible relación negativa entre el color y la elaboración del canto de las aves, en el caso de aves con presupuesto energético limitado para invertir en la comunicación. Otro parámetro muy importante que determina la estructura de las señales es la depredación, pero esta es muy difícil de medir directamente en campo. Las relaciones negativas observadas desde hace un par de siglos, pueden deberse a que diferentes canales de comunicación funcionan mejor en ciertos hábitats, cada uno de los cuales pueden determinar estrategias diferentes para lograr una comunicación efectiva.

\section{Palabras clave}

Bioacústica, señales visuales, comunicación, aves. 


\section{SIMPOSIO: CAFÉ, CACAO Y AVES \\ UTILIZANDO EPIFITAS PARA AUMENTAR LA DIVERSIDAD DE AVES EN UNA FINCA DE CAFÉ}

\section{Ernesto M. Carman}

Get Your Birds!, 1-7100 Paraíso, 30201, Costa Rica.

E-mail: emcarman@gmail.com

El cultivo de café bajo sombra beneficia muchas especies de aves. En esta investigación se demuestra cómo se puede incrementar la cantidad de especies y/o individuos de aves que utilizan una plantación de café al propagar manualmente especies nativas de epifitas en los árboles de sombra. La propagación de epifitas se podría utilizar como parte de un sistema integrado de control de plagas al crear un ecosistema más atractivo para especies que controlan la broca del café, como aves, murciélagos y ranas. De igual manera, se provee de hábitat a una gran cantidad de especies.

Palabras clave

Aves, café bajo sombra, manejo, control de plagas.

\section{AVES ASOCIADAS A SISTEMAS AGROFORESTALES DE CAFÉ EN LA CUENCA DEL MAYO- CHINCHIPE, SURESTE DE ECUADOR}

\section{Leonardo Ordóñez-Delgado}

Departamento de Ciencias Biológicas, Universidad Técnica Particular de Loja, San Cayetano Alto, Calle París, Loja, Ecuador.

Email: 1yordonez2@utpl.edu.ec

La producción cafetalera constituye una de las principales actividades agropecuarias del sur del Ecuador. Ésta posee la particularidad de desarrollarse mediante principios de agroforestería; es decir, combinando árboles nativos que proveen beneficios de diverso tipo a los cafetales. Esta situación determina que estos sistemas productivos sean importantes para la biodiversidad local, principalmente para las aves. A pesar de que mucho del café comercializado de estos sitios se presenta como café "ecológico, verde o amigable con la naturaleza", el conocimiento sobre su fauna asociada es aún básico. Son pocos los trabajos existentes sobre las aves presentes en los cafetales del país, ninguno de ellos abarca el sur de Ecuador. En febrero y marzo de 2016 efectuamos una caracterización de aves asociadas a cafetales de diferente tamaño y composición arbórea en Zumba y Palanda, sur de Zamora Chinchipe. Registramos 100 especies de aves asociadas a los cafetales, entre las que se cuentan cuatro especies endémicas (dos tumbesinas y dos del valle del Marañón), una especie casi amenazada a nivel global y nacional, y tres migratorias boreales. Al menos cuatro especies se encontraban anidando dentro de los cafetales. Todas las especies utilizaban elementos de este agroecosistema para diversos procesos ecológicos: búsqueda de alimento, material para nidos, construcción de nidos o para movilizarse entre zonas boscosas. Si bien esta es una primera aproximación que evidencia la importancia de los cafetales para las aves de la región -y la biodiversidad local en general-, es fundamental desarrollar estudios que permitan conocer las dinámicas que se desarrollan en los cafetales, con la finalidad de fortalecer esta actividad como ejemplo local para mitigar el impacto de actividades antrópicas negativas para la biodiversidad.

\section{Palabras clave}

Aves, conservación, cafetales arbolados, Zamora Chinchipe. 


\title{
AVIFAUNA ASOCIADA A CACAOTALES MANEJADOS Y ABANDONADOS EN EL CARIBE DE COSTA RICA
}

\author{
Daniel Martínez-A \\ Apartado Postal 497-7050, Cartago, Costa Rica. \\ E-mail: jacamerops@yahoo.com
}

En los paisajes agrícolas se puede encontrar una cantidad significativa de área reforestada formada por sistemas agroforestales manejados, cuyas características estructurales complejas, el almacenamiento de microclimas y la diversidad de plantas en el dosel sirven de alimento y contribuyen a la existencia de una biota importante. En este trabajo describo la avifauna asociada a distintos cacaotales manejados, así como la de algunos cacaotales con 45 años de abandono, que se encuentran en proceso de sucesión, en elevaciones bajas e intermedias del Caribe sur y Caribe central de Costa Rica. Los datos fueron tomados mediante conteos por puntos fijos en varios estudios, así como captura con redes de niebla y observaciones casuales. Destaco principalmente algunas especies migratorias de las familias Tyrannidae, Turdidae y Parulidae, consideradas como raras, poco comunes o amenazadas; también la presencia de algunas especies residentes poco comunes que requieren de este tipo de hábitat para sobrevivir. En los cacaotales abandonados en sucesión registré un total de 146 especies, mientras que cacaotales comerciales registré un total de 75 especies. Destacan algunas especies como Micrastur mirandollei, Cyanocorax affinis, Catharus fuscescens, Vermivora chrysoptera, Setophaga caerulescens y Cardellina canadensis, aunque también registré varias especies más comunes. Los cacaotales sirven tanto de refugio como para alimentación, anidamiento y descanso para una alta diversidad de especies, algunas de ellas en estado conservación crítico. Por esto, es necesario promover la protección e investigación de aves en estos hábitats todavía poco estudiados, tanto en cacaotales que existen en Costa Rica como en Ecuador, que comparten características climáticas y de composición de especies.

\section{Palabras clave}

Agroforestería, avifauna, biodiversidad, cacaotales, conservación, aves migratorias.

\section{AVANCES PARA LA CERTIFICACIÓN DEL CACAO AMIGABLE CON LAS AVES: LA EXPERIENCIA EN LA REGIÓN DEL CHOCÓ CON LOS PEQUEÑAS PLANTACIONES DE CACAO}

\author{
Manuel Sánchez Nivicela \\ Email: clandestine.bird@gmail.com
}

Existe un importante potencial económico y de conservación que podría ayudar a las comunidades de pequeños productores de cacao que aún subsisten dentro de áreas con cobertura vegetal importante para aves endémicas y amenazadas del área de endemismo del Chocó. Durante salidas de campo llevadas a cabo en diciembre de 2010 se pudo notar que, en un principio, las ideas de certificación amigable con las aves migratorias de Norteamérica pueden proponerse como una alternativa más abierta para proteger especies residentes. Se registraron aves endémicas del Chocó y globalmente amenazadas en pequeñas plantaciones de cacao en Playa de Oro, en la provincia de Esmeraldas. Asimismo, durante la investigación se analizó el beneficio de la vegetación que brinda sombra al cacao, encontrando un límite que puede beneficiar económicamente a los pequeños productores, así como impulsar la producción y consumo responsable amigable con los ecosistemas.

\footnotetext{
Palabras clave

Cacao, agroforestería, certificación, conservación.
} 


\title{
RESTAURACIÓN ECOLÓGICA Y CONSERVACIÓN DE AVES EN LA MANCOMUNIDAD DEL CHOCÓ ANDINO: RESERVA MASHPI SHUNGO, PACTO, PICHINCHA
}

\author{
Alejandro Solano-Ugalde \\ Fundación Imaymana, Paltapamba 476, San Pedro del Valle, Nayón, Quito, Ecuador. \\ E-mail: jhalezion@gmail.com
}

El presente estudio fue realizado en la Reserva Mashpi Shungo, parroquia Pacto, provincia de Pichincha. En la reserva se investigó la comunidad de aves existente en cuatro parcelas destinadas a la restauración ecológica mediante forestería análoga, comparando resultados del año inicial y cinco años más tarde. La comunidad de aves registrada antes del inicio de las actividades de restauración fue de 52 especies de aves. Cinco años más tarde se evidenció un aumento en el número de especies hasta 156. Este importante cambio en la comunidad de aves es discutido y se concluye que entre lo más relevante del estudio es la necesidad de que más campesinos y campesinas adopten la forestería análoga, así como seguir monitoreando las aves en distintos tipos de manejo y uso del suelo. Se presenta una metodología para un estudio a realizarse a través de un convenio con la universidad de Cornell y el programa regional PROALAS. Se comparte esta herramienta para que más investigadores y observadores de aves apoyen en la recolección de datos para alimentar proyectos de mayor envergadura en favor de posibles escenarios de certificación de productos amigables con el ambiente, que den un valor agregado a la producción agrícola sostenible.

\section{Palabras clave}

Aves, Chocó, restauración, producción, conservación. 


\title{
PLANTAS CLAVE PARA CONSERVAR LAS FUNCIONES DE LAS AVES EN LOS ECOSISTEMAS ANDINOS DEL SUROESTE DEL ECUADOR
}

\author{
Juan M. Aguilar*, Andrea Nieto, Antonio Crespo, Boris A. Tinoco \\ Escuela de Biología, Ecología y Gestión, Universidad del Azuay, \\ Av. 24 de Mayo 7-77 y Hernán Malo, Cuenca, Ecuador. \\ *Autor para correspondencia; E-mail: juanmaguilaru@yahoo.com
}

Las interacciones interespecíficas mantienen la estabilidad estructural de las comunidades biológicas y las interdependencias mutualistas pueden brindar una perspectiva comunitaria para la conservación de las especies. El análisis de redes de interacción puede resaltar diferentes características de una comunidad, destacando atributos esenciales para mantener la diversidad y conectividad de la red. En una comunidad, las especies se relacionan de manera diferente. Algunas especies generalistas que comparten muchos vínculos y están muy conectadas, muestran importancia desproporcionada dentro de la red de interacciones y son clave para mantener o restaurar las funciones de los ecosistemas. Basándose en observaciones de campo en cuatro diferentes ecosistemas andinos del suroeste del Ecuador, en la provincia de Azuay, obtuvimos índices estructurales de las redes de interacción entre plantas y colibríes. Estos índices permitieron identificar localmente las plantas más importantes para mantener las funciones ecológicas y la diversidad de colibríes. Este método sencillo permitió establecer que especies como Oreocallis grandiflora y Macleania rupestris constituyen recursos clave en escala regional, al mostrar importancia constante entre ecosistemas con diferente composición de interacciones. Estas especies deben considerarse clave en futuros objetivos de restauración de ecosistemas, conservación e investigación dentro de los Andes del sur del Ecuador.

Palabras clave

Comunidad biológica, funciones de los ecosistemas, red de interacciones, restauración ecológica.

\section{AVES TERRESTRES DE GALÁPAGOS: ESTATUS, AMENAZAS Y DÉFICIT DE CONOCIMIENTO}

\section{David Anchundiaa ${ }^{*}$, Jorge Carrión ${ }^{2}$, Michael Dvorak ${ }^{3}$, Denis Mosquera', Erwin Nemeth ${ }^{3}$, Christian Sevilla' ${ }^{2}$, Beate Wendelin ${ }^{3}$, Birgit Fessl ${ }^{1}$}

'Fundación Charles Darwin, Puerto Ayora, Galápagos, Ecuador.

2Dirección del Parque Nacional Galápagos, Puerto Ayora, Galápagos, Ecuador.

${ }^{3}$ BirdLife Austria.

*Autor para correspondencia; E-mail: davidanchundia@gmail.com

En las islas Galápagos, la presión humana se ha incrementado permanentemente desde hace más de un siglo, lo que ha repercutido en la necesidad de proponer acciones de conservación que, para ser exitosas, requieren de un adecuado conocimiento de las especies que se desea proteger. A pesar que la avifauna de Galápagos se considera bien estudiada, poco se sabe de la abundancia de sus especies, por lo que en algunas se ha notado un grave declive en sus tamaños poblacionales. La Fundación Charles Darwin, la dirección del Parque Nacional Galápagos y varios colaboradores están levantando información de línea base con un método estandarizado para reevaluar el estado de la avifauna, identificar amenazas y proponer soluciones. Presentamos un resumen de los resultados de monitoreo efectuados hasta 2016 en cinco islas: San Cristóbal, Santa Cruz, Floreana, Santiago e Isabela. Las especies más afectadas son la Paloma de Galápagos Zenaida galapagoensis, con muy pocas observaciones en tres de cinco islas; el Pinzón Grande de Árbol Camarhynchus psittacula, ausente de Floreana y con números bajos en Santa Cruz, Isabela y Santiago. Por su parte, el Pájaro Brujo Pyrocephalus nanus está en estado crítico en Santa Cruz y ausente en Floreana y en la parte alta de Santiago, mientras que otra especie de Pájaro Brujo (P. dubius) al parecer ha desaparecido de San Cristóbal, lo que constituiría en la primera especie galapagueña extinta en tiempos modernos. Dos especies amenazadas según UICN, el Cucuve de San Cristóbal Mimus melanotis y el Pinzón Mediano de Árbol Camarhynchus pauper están en mejor 
estado de lo que se pensaba. Para especies como la Golondrina de Galápagos Progne modesta se debe buscar otras formas de monitoreo involucrando la comunidad de guías, locales y turistas. Todavía falta mucho para una cobertura completa del archipiélago, pero se confirman las tendencias fuertes de disminución de aves.

\title{
Palabras clave
}

Disminución poblacional, línea base, estado de conservación, Galápagos.

\section{PATRONES DE DIVERSIDAD DE AVES EN LAS ORILLAS DE RÍOS TOMEBAMBA Y YANUNCAY, CUENCA}

\author{
Victoria Argudo*, María Cristina Ríos \\ Universidad del Azuay, Av. 24 de Mayo 7-77 y Hernán Malo, Cuenca, Ecuador. \\ *Autora para correspondencia; E-mail: vicky.argudo19@gmail.com
}

Analizamos la diversidad y preferencia de hábitat de las comunidades de aves en 50 puntos a lo largo de los ríos Tomebamba y Yanuncay, ciudad de Cuenca, con la finalidad de determinar si existe variación en la diversidad y uso de hábitat bajo la influencia del paisaje urbano. Identificamos y contabilizamos 4739 individuos correspondientes a 45 especies, 22 familias de aves y 10 órdenes. Las familias con mayor número de especies fueron Trochilidae (6 especies), seguida de Columbidae, Thraupidae y Tyrannidae (4 especies) y Emberizidae (3 especies). Las especies más abundantes fueron la Golondrina Azuliblanca Notiochelidon cyanoleuca, el Chingolo Zonotrichia capensis, Mirlo Chiguanco Turdus chiguanco, Paloma Doméstica Columba livia y Orejivioleta Ventriazul Colibri coruscans, todas consideradas especies tolerantes a áreas antropizadas. En cuanto a las interacciones, el análisis identificó que las especies vegetales con mayor número de interacciones son Salix humboldtiana, la cual interactúa con 4-7 especies de aves, seguida de Eucalyptus globulus con 4-5 especies y Prunus serotina con 3-5 especies. Los resultados indican que la riqueza y abundancia de la avifauna en las orillas de los ríos es diferente entre los tramos estudiados; sin embargo, esta diferenciación no está influenciada por las variables de cobertura del paisaje. Aunque en los análisis de redes de interacción las especies arbóreas mencionadas aparecen como las más significativas para forrajeo en los tres tramos de estudio, estas especies no son necesariamente las más aptas o las únicas idóneas para planes de mantenimiento de las riberas. La vegetación original de ribera ha sido destruida o reemplazada por especies exóticas, con pocos remanentes de vegetación original. Es fundamental implementar el manejo de las orillas enfocadas a la conservación, aumento y estudio de la biodiversidad urbana como medio de convivencia del ser humano con su entorno.

Palabra clave

Aves urbanas, interacción, paisaje urbano, sistema ribereño.

\section{POSIBLE DESPLAZAMIENTO ENTRE EL GAVILÁN VARIABLE GERANOAETUS POL YOSOMA Y EL GAVILÁN ALICASTAÑO PARABUTEO UNICINCTUS EN LOS VALLES INTERANDINOS AL NORTE DEL ECUADOR}

\section{Josué D. Arteaga Torres*, Tjitte de Vries}

Ecología y Avifauna, Facultad de Ciencias Exactas y Naturales, Pontificia Universidad Católica del Ecuador, Quito, Ecuador.

*Autor para correspondencia; E-mail: josue.arteaga.t@gmail.com

Aunque existe poca información sobre la población del Gavilán Alicastaño Parabuteo unicinctus en el Ecuador, creemos que existe un aumento poblacional en la región interandina. Planteamos como hipótesis el posible desplazamiento del Gavilán Variable Geranoaetus polyosoma en los valles interandinos por parte de $P$. unicinctus a causa del cambio de uso de suelo y efectos del cambio climático. En varias salidas de campo durante los últimos 5 años no hemos observado nidos activos o abandonados de G. polyosoma. Mediante bases de datos globales obtuvimos un total de 147 registros históricos de P. unicinctus y 59 de G. polyosoma, 
con los cuales determinamos las zonas de conflicto en el área muestreo mediante modelamiento de nicho con el software Maxent. Muestreamos 39 territorios potenciales $\left(28 \mathrm{~km}^{2}\right)$ entre noviembre 2015 y marzo 2016 en los valles interandinos de Pichincha e Imbabura. Visitamos cada sitio cuatro veces y generamos la descripción vegetal y de uso de suelo para cada territorio. Obtuvimos un total de 1840 datos climáticos y 550 registros de aves rapaces diurnas y nocturnas. Mediante análisis multivariados determinamos las relaciones que existen entre las diferentes especies observadas y las variables climáticas y de vegetación de cada punto de muestreo. Encontramos una alta relación entre el uso de suelo degradado (zonas rurales, periurbanas y agrícolas) y la presencia de $P$. unicinctus. Debido a que solo obtuvimos un registro de $G$. polyosoma durante los 5 meses de muestreo, proponemos un mayor muestreo a nivel temporal y espacial para determinar con mayor precisión la situación poblacional de G. polyosoma en los valles interandinos. Con los registros obtenidos en el campo y los datos históricos generamos una serie de mapas con proyecciones a climas futuros y pasados para estimar el cambio en la distribución de las especies de estudio.

Palabras clave

Competencia interespecífica, conservación, modelamiento de nicho, Parabuteo unicinctus, Geranoaetus polyosoma, Andes.

\title{
EVALUACIÓN DE LOS IMPACTOS DE LA GANADERÍA EN LAS AVES DE PÁRAMO EN LOS ANDES SUR DEL ECUADOR
}

\section{Pedro X. Astudillo, Santiago Barros*, David C. Siddons, Edwin Zárate}

Universidad del Azuay, Escuela de Biología, Ecología y Gestión, Av. 24 de Mayo 7-77, Cuenca, Ecuador.

*Autor para correspondencia; E-mail: jsanty.b1@gmail.com

El pastoreo en pastizales naturales es extenso y con negativas consecuencias para la biodiversidad. En los altos Andes, el páramo herbáceo es un ecosistema distintivo en donde la influencia del pastoreo es pobremente documentada. Evaluamos la influencia del páramo modificado por actividades ganaderas sobre grupos de hábitat de aves en los Andes sur del Ecuador. Estudiamos tres localidades con diferentes niveles de pastoreo mediante transectos. Encontramos disminución en las aves especialistas en páramos homogéneos alterados. Esta pérdida de cobertura vegetal puede representar menos hábitat clave para la sobrevivencia de aves especialistas.

Palabras clave

Abundancia, altitud, especialistas de páramo, gremios de hábitat.

\section{COMPARACIÓN DE DOS METODOLOGÍAS DE OBSERVACIÓN DE FRUGIVORÍA EN EL BOSQUE HÚMEDO TROPICAL DEL CHOCÓ, ESMERALDAS, ECUADOR}

\author{
Domingo Cabrera', Michael Mahoney ${ }^{2}$, Jorge Olivo', Luke Browne ${ }^{1,2}$, Jordan Karubian ${ }^{1,2^{*}}$ \\ 'Fundación para la Conservación de los Andes Tropicales (FCAT), Mariano Hurtado N 50-89 y Vicente \\ Heredia, Quito, Ecuador. \\ ${ }^{2}$ Department of Ecology \& Evolutionary Biology, Tulane University, 400 Boggs Building, New Orleans, LA, \\ 70118, Estados Unidos. \\ *Autor para correspondencia; E-mail: jordankarubian@gmail.com
}

En las áreas tropicales muchos árboles dependen de la dispersión de sus semillas por animales (zoocoria) y las diferencias en las especies que consumen frutos tienen importantes implicaciones para su reproducción. Los objetivos de este estudio fueron determinar si existen diferencias entre las aves diurnas que consumen las frutas de tres especies de árboles, y presentar dos metodologías para encontrar dispersores primarios de árboles adultos con fruta en el bosque húmedo tropical del Chocó, en el noroeste de Ecuador. Presentamos datos de más de 680 horas de observación entre tres especies con fruta en nuestro sitio de estudio, las palmas Bactis setulosa y Oenocarpus bataua, y la Myristicaceae Virola dixonii. Entre las dos especies de palma y la 
especie de Virola encontramos nueve dispersores de semillas; cinco dispersores primarios con tasas de dispersión altas, tales como tucanes (Ramphastidae) y una especie de cotinga, Cephalopterus peduliger. También presentamos los datos de nuestras trampas cámara colocadas en individuos de $O$. bataua. Entre las dos metodologías encontramos que mientras que con la observación directa del árbol observamos tasas más altas de consumo de semillas y fruta, con las trampas cámara encontramos mayores cantidades de mamíferos diurnos visitando los árboles. Adicionalmente, podemos acotar que con las trampas cámara pudimos identificar dispersores que visitan los árboles por la noche, como el caso del cusumbo Potos flavus.

Palabras clave

Aves frugívoras, Cotingidae, observación de palmas, trampas cámara, Ramphastidae.

\section{LISTADO DE AVIFAUNA DEL PARQUE NACIONAL YASUNÍ}

\section{Héctor Cadena-Ortiz*, Patricia Bejarano-Muñoz}

Unidad Técnica de Apoyo al Parque Nacional Yasuní, Programa de Reparación Ambiental y Social (PRAS), Ministerio del Ambiente (MAE), Av. Amazonas N24-196 y Cordero, Edificio Contempo, Quito, Ecuador. *Autor para correspondencia; E-mail: fercho_cada@yahoo.es

El Sistema Nacional de Áreas Protegidas, con 51 reservas naturales, cubre el 20\% de la superficie del Ecuador. El Parque Nacional Yasuní (PNY), con 1022736 ha es el área protegida más grande del Ecuador continental, reconocida por su alta biodiversidad, incluso a escala mundial. El PNY está dentro del Área de Importancia para las Aves Gran Yasuní. Sin embargo, esta área protegida no cuenta con un inventario formal de su ornitofauna. Se sabe que los bosques del piso húmedo oriental en la Amazonía ecuatoriana albergan más de 694 especies de aves. Para el PNY hay listados publicados de sitios puntuales: 319 especies de aves en la Estación de Biodiversidad Tiputini; 420 especies en la Estación Científica Yasuní; 205 en los alrededores de la comunidad waorani Guiyero. Hay también varios informes de consultorías dentro del PNY, principalmente ligados a la actividad hidrocarburífera. Entre ellos, podemos mencionar el registro de 491 especies de aves en el Bloque 16 al noroeste del parque. El conocimiento certero de la riqueza biótica permite tomar decisiones más acertadas en pro de la conservación. Para generar una lista completa de aves del PNY, estamos analizando las publicaciones existentes, bases de datos en línea y especímenes de museos. A la par, acudimos a especialistas que han trabajado dentro del PNY con este grupo faunístico. Un listado preliminar indica que el PNY alberga al menos 656 especies de aves, agrupadas en 21 órdenes y 63 familias; 12 especies amenazadas a nivel global y 17 a nivel nacional. Como en otros bosques lluviosos de tierras bajas neotropicales, predominan representantes de la familia Tyrannidae (13\%) y Thamnophilidae (10\%). Existen áreas poco muestreadas, principalmente al límite sur del PNY, a donde se han planificado varias expediciones para este año.

\section{Palabras clave}

Amazonía, avifauna, conservación, especies amenazadas, listados, Yasuní.

\section{EGAGROPILAS DE BÚHOS, RAUDAL DE INFORMACIÓN}

\section{Héctor Cadena-Ortiz ${ }^{1,2, *}$, Jorge Brito M.2}

'Programa de Reparación Ambiental y Social (PRAS), Ministerio del Ambiente, Av. Amazonas N24-196 y Cordero. Edificio Contempo, Quito, Ecuador.

${ }^{2}$ Museo Ecuatoriano de Ciencias Naturales del Instituto Nacional de Biodiversidad. Rumipamba 341 y Av. de los Shyris, Casilla: 17-07-8976, Quito, Ecuador.

*Autor para correspondencia; E-mail: fercho_cada@yahoo.es

El conocimiento sobre aves rapaces nocturnas ecuatorianas es escaso, en particular la información trófica a nivel temporal y espacial. La comprensión de la dieta es importante para entender la función que cada especie cumple en su medio, qué organismos depreda y cuáles son sus requerimientos energéticos. Una alternativa no invasiva para estudiar la dieta es el análisis de sus egagrópilas, que son restos regurgitados que contienen las 
partes indigeribles de sus presas, como pelos, plumas y huesos. El análisis de egagrópilas es simple: una vez localizado el comedero del ave, no se requiere de muchos insumos. Las egagrópilas colectadas pueden ser desmenuzadas en seco o humedeciéndolas con agua jabonosa. Es importante precautelar la seguridad del investigador protegiéndose con guantes y mascarilla al momento del análisis; los restos de roedores en las egagrópilas podrían conservar virus o bacterias. El análisis de egagrópilas provee información de la dieta de la rapaz, y permite conocer su radio de caza, registros interesantes de sus presas, que en algunas ocasiones son especies difíciles de capturar con métodos de muestro tradicionales. También se puede inferir el estado poblacional de las presas y su variación estacional con colectas periódicas de egagrópilas. Existen artículos publicados de contenido de egagrópilas en Ecuador acerca de Tyto alba, Ciccaba albitarsis y Asio flammeus galapagoensis. Está en prensa información sobre Athene cunicularia y y A. flammeus bogotensis, y hay artículos en preparación sobre Bubo virginianus y Asio stygius. Una de las mayores dificultades en los estudios de dieta es la ausencia de datos para la identificación a partir de fragmentos de presas consumidas. Esta dificultad se agrava en ecosistemas con alta diversidad como en Ecuador, por lo que es necesario continuar buscando egagrópilas, colectándolas, analizándolas y publicando su información.

\title{
Palabras clave
}

Dieta, diversidad, registros, regurgito, Strigidae, Tytonidae.

\section{IMPACTO DEL CAMBIO CLIMÁTICO SOBRE LA DISTRIBUCIÓN POTENCIAL DEL PERICO PECHIBLANCO PYRRHURA ALBIPECTUS}

\author{
Marlon Cobos ${ }^{1, *}$, Claudia Nuñez ${ }^{1}$, Christian Mendoza ${ }^{2}$, Katiusca Valarezo ${ }^{2}$ \\ 'Facultad de Biología, Universidad de La Habana, Cuba. \\ 2Zoológico Municipal de Loja, Ecuador. \\ *Autor para correspondencia; E-mail: manubio13@gmail.com
}

El cambio climático es actualmente una de las principales amenazas para la biodiversidad. Se considera que las especies más vulnerables son las de menor distribución y menor amplitud de nicho. El Perico Pechiblanco Pyrrhura albipectus, como especie endémica del sur de Ecuador y norte de Perú, podría ser vulnerable a este fenómeno. No obstante, esta amenaza no tiene un comportamiento homogéneo en la región y aún no se ha realizado este análisis para la especie. Por ello, nuestro objetivo fue identificar los impactos del cambio climático sobre la distribución potencial del Perico Pechiblanco. Para esto, modelamos su distribución potencial actual y la proyectamos al año 2050, en dos escenarios de emisión (RCP2.6 y RCP8.5) y tres modelos de circulación general. En los modelos resultantes se identificaron las áreas adecuadas y no adecuadas para la especie. Se realizó un consenso entre los resultados y se identificaron las áreas adecuadas para la especie que se conservarían, las que se perderían y las nuevas áreas adecuadas en el futuro. Las áreas actuales se extienden más que la distribución propuesta, pero las áreas que mejor se conservarían en el futuro son las cercanas a los registros actuales. El escenario RCP8.5 representaría mayores pérdidas de áreas adecuadas. En los dos escenarios se presentarían nuevas áreas adecuadas, con mayor cantidad en el RCP2.6. Del total de poblaciones, un 14,3\% para el escenario RCP2.6 y un 21,4\% para el RCP8.5 se ubican en zonas que perderían las condiciones adecuadas. Las poblaciones de esta especie que permanezcan en las zonas de adecuación que se perderían en el futuro podrían decaer en su estado de conservación, por lo que su respuesta podría ser el desplazamiento. Entender el cambio espacial de las condiciones climáticas para esta especie podría incentivar la propuesta de medidas de conservación adecuadas en un entorno cambiante.

\section{Palabras clave}

Calentamiento global, cambio de distribución, disminución poblacional, Pyrrhura albipectus. 


\title{
BUTEO POL YOSOMA Y BUTEO POECILOCHROUS SON DOS ESPECIES DISTINTAS, PUNTO FINAL
}

\author{
Tjitte de Vries ${ }^{1, *}$, José Cabot ${ }^{2}$ \\ 'Escuela de Ciencias Biológicas, Pontificia Universidad Católica del Ecuador, Quito, Ecuador. \\ ${ }^{2}$ Estación Biológica Cota Doñana, Sevilla, España. \\ *Autor para correspondencia; E-mail: tdevries@puce.edu.ec
}

\begin{abstract}
¿Por qué continúa la controversia sobre si estos gavilanes son clasificados como co-específicos o pertenecientes a dos especies diferentes? Para que el Ecuador no pierda una especie indicamos que: 1) las dos especies comparten un plumaje con dos morfos de coloración: oscuro y claro; 2) son sexualmente dimórficas; 3) las diferencias en dimensiones y proporciones hace que tengan distintos tamaños y siluetas, sin que se produzca solapamiento en algunas dimensiones corporales; 4) presentan patrones de plumaje semejantes, especialmente las aves del morfo pálido; 5) sin embargo, difieren en patrones de plumaje del morfo oscuro; 6) el plumaje definitivo se alcanza mediante un ciclo de cambios durante seis años; 7) tienen distinta carga alar sin formas intermedias; 8) existen también diferencias en el patrón de distribución geográfica en las aves de mayor talla: Buteo polyosoma está en el extremo sur de Sudamérica y B. poecilochrous en Colombia y Ecuador; 9) en ningún caso se puede considerar a B. poecilochrous como una subespecie de B. polyosoma; de las investigaciones realizadas, se sabe que $B$. poecilochrous tiene dos subespecies reconocidas $B$. $p$. poecilochrous y B. p. fjeldsai; 10) el proceso evolutivo de los dos taxones va por rutas distintas; 11) existe diferenciación en el hábitat de anidación de las dos especies; 12) no reconocer las dos especies causa problemas de conservación para B. polyosoma en los Andes del Ecuador; 13) su reciente especiación ha hecho que las diferencias genéticas no sean detectables; por ello, los estudios sobre su morfología y forma de vida independientes han aportado mayor información; 14) en base al concepto biológico de especies, las diferencias morfológicas y de forma de vida confirman que las dos especies son distintas y para incentivar la conservación se debe iniciar con el reconocimiento de B. polyosoma y B. poecilochrous. Actualmente, los dos gavilanes son clasificados en el género Geranoaetus, junto con G. melanoleucus y G. albicaudatus.
\end{abstract}

\section{Palabras clave}

Buteo poecilochrous, Buteo polyosoma, clasificación, conservación, dimorfismo sexual, especies distintas, morfos.

\section{TODO LO QUE TODAVÍA NO CONOCEMOS SOBRE LAS AVES DEL ECUADOR}

\author{
Juan Freile \\ Red Aves Ecuador y Comité Ecuatoriano de Registros Ornitológicos, Pasaje El Moro E4-216 y Norberto \\ Salazar, Tumbaco, Ecuador. \\ E-mail: jfreileo@yahoo.com
}

Más de 150 años, más de 1500 investigadores, más de 4000 estudios publicados y unas cuantas monografías sobre las aves del Ecuador y de Galápagos (por separado) no han alcanzado para comprender en su totalidad la ecología, distribución, conservación y sistemática de nuestra avifauna. Cada nueva investigación descubre algo que desconocíamos: una especie fuera de su rango de distribución, un nido nuevo, relaciones filogenéticas insospechadas, poblaciones en declinación. Sin importar cuánto esfuerzo hagamos ni cuánto tiempo invirtamos, nunca lograremos conocerlo todo. Tenemos 1679 especies por estudiar y nuestra comunidad ornitológica todavía está madurando. Aunque Ecuador está entre los diez países neotropicales con mayor conocimiento ornitológico, países como Brasil, Argentina o México nos superan hasta cinco veces en número de publicaciones -más aún en número de ornitólogos, instituciones de investigación y recursos. Hacia 2004, un $75 \%$ de las aves del Ecuador no contaban con estudios publicados, generados en el país. En la última década ese porcentaje disminuyó a 40-45\%. Sin embargo, este incremento no debe confundirnos: son recién las primeras publicaciones de unas 500 especies. El conocimiento sigue siendo limitado. Conocer un nido o reportar un aspecto del comportamiento de una especie está muy lejos de comprender su ecología. Desde 2013, el Comité Ecuatoriano de Registros Ornitológicos ha reportado 13 especies nuevas para Ecuador, y cada 
año se publican nuevos registros geográficos. La distribución de las aves dista de comprenderse bien; más todavía los patrones migratorios o la biogeografía de las especies. Incluso en Galápagos, famoso laboratorio natural de la evolución, hay especies muy poco conocidas como los pájaros brujos Pyrocephalus dubius y $P$. nanus, el Pachay Laterallus spilonota o el Petrel de Tormentas Oceanites gracilis. Aunque Ecuador parece no ser fuente de especies nuevas, tan anheladas entre los ornitólogos, no es descabellado sospechar que aún tenemos algún gran misterio esperando ser descubierto.

\title{
Palabras clave
}

Conocimiento, conservación, distribución, ecología, Ecuador, investigación, sistemática.

\section{PATRONES DE DIVERSIDAD DE AVES DE LA PROVINCIA DE EL ORO}

\author{
César Garzón Santomaro',", Patricio Mena Valenzuela1, Francisco Sornoza ${ }^{2}$, Glenda Pozo-Zamora1, \\ Gabriela Echeverría-Vaca ${ }^{1}$ \\ ${ }^{1}$ Museo Ecuatoriano de Ciencias Naturales del Instituto Nacional de Biodiversidad (MECN-INB), \\ Rumipamba 341 y Av. De los Shyris, Quito, Ecuador. \\ ${ }^{2}$ Fundación de Conservación Jocotoco, Lizardo García E9-104 y Andrés Xaura, Quito, Ecuador. \\ *Autor para correspondencia; E-mail: cesar.garzon@ambiente.gob.ec
}

La provincia de El Oro contiene un mosaico ecosistémico en un gradiente altitudinal que va desde el nivel del mar hasta $3900 \mathrm{~m}$ en los Andes occidentales, donde existen 17 sistemas ecológicos distintos. Estudiamos 17 localidades mediante recorridos de observación en diferentes periodos del día para cubrir especies diurnas y nocturnas. Para el registro de aves acuáticas realizamos recorridos de observación en una canoa y al borde de los cuerpos de agua. Registramos 556 especies de 347 géneros y 71 familias. Las familias más diversas fueron Tyrannidae (66 especies), Thraupidae (55) y Trochilidae (46). Identificamos 48 especies restringidas a cuatro centros de endemismo, siendo las más importantes Pyrrhura orcesi y Scytalopus robbinsi, endémicas de Ecuador. Registramos 59 especies migratorias, 42 de ellas provenientes del hemisferio norte. Además, encontramos 19 especies clasificadas en alguna categoría de amenaza. La curva de acumulación de especies no muestra una tendencia de estabilización en su asíntota, por lo que se estima un incremento de hasta $27 \%$ en la diversidad. El patrón de diversidad de aves en función de los biomas en la provincia de El Oro muestra picos máximos de riqueza de especies en el bioma piemontano (400-1600 m s.n.m.). La riqueza disminuye a medida que se aleja de esta banda de elevación. Los ecosistemas piemontanos muestran una mayor remanencia de bosques en la provincia. En ellos confluyen especies de tierras bajas y especies andinas. Los bosques secos de tierras bajas y las zonas de manglar son los siguientes ecosistemas en cuanto a diversidad. Se hace necesaria la conservación de varios remanentes boscosos, en especial bosques piemontanos y secos, amenazados por actividades mineras, ganaderas y agrícolas.

\section{Palabras clave}

Bosque piemontano, bosque seco, diversidad, endemismo, manglar, El Oro.

\section{RESPUESTAS DE LAS REDES DE POLINIZACIÓN AVE-PLANTA AL DISTURBIO AMBIENTAL EN BOSQUES ALTO-ANDINOS DE PICHINCHA, ECUADOR}

\author{
Esteban Guevara ${ }^{1,3^{*}}$, Bryan Rojas ${ }^{1,2}$, Cristian Poveda ${ }^{1,2}$, Tatiana Santander ${ }^{3}$ \\ 1 Área de Investigación y Monitoreo de Avifauna, Aves y Conservación - BirdLife en Ecuador, Pasaje \\ Joaquín Tinajero E3-05 y Jorge Drom, Quito, Ecuador. \\ ${ }_{2}^{2}$ Escuela de Ciencias Biológicas, Pontificia Universidad Católica del Ecuador, Av. 12 de Octubre 1076 y \\ Roca, Quito, Ecuador. \\ *Autor para correspondencia; E-mail: eguevara@avesconservacion.org
}

Los bosques alto-andinos del noroccidente de Ecuador han sufrido históricamente una marcada pérdida y degradación debido a usos agropecuarios principalmente. En este contexto, la posible pérdida de especies de aves y plantas en distintos niveles de disturbio podría afectar también las interacciones ecológicas en las que 
dichas especies están involucradas, afectando en última instancia a procesos ecológicos como la polinización. Nuestro estudio se enfocó en estudiar las respuestas de redes de polinización planta-ave a un gradiente de disturbio ambiental. Para ello, identificamos cinco tipos de hábitats con distintos niveles de degradación. Seguidamente, en cada hábitat identificamos las plantas con síndrome ornitófilo, frente a las cuales colocamos cámaras de vigilancia a una distancia de entre $80-100 \mathrm{~cm}$. Las cámaras se programaron para obtener una foto cada segundo durante 11 horas diarias, entre 06h00-18h30, con una pausa de $30 \mathrm{~min}$ al medio día. Las imágenes obtenidas se analizaron con una herramienta de detección de movimiento (Motion Meerkat) para identificar las fotos en las cuales se capturaron interacciones entre plantas y aves. Analizamos la respuesta de las redes de polinización utilizando técnicas de análisis de redes bipartitas (de dos niveles tróficos). En general, nuestros resultados muestran que la organización de las redes de polinización cambió en los distintos hábitats estudiados. Las redes de polinización planta-ave en hábitats menos degradados exhiben una estructura más estable que se refleja en atributos topológicos como el número posible de interacciones y el anidamiento (patrón en el cual las especies especialistas interactúan con un subgrupo de especies generalistas). Adicionalmente, algunas plantas difieren en su capacidad de responder a disturbios ambientales, siendo algunas capaces de sostener interacciones con aves en hábitats más degradados. Finalmente, algunas especies de colibríes generalistas como el Metalura Tiria Metallura tyrianthina son importantes polinizadores en hábitats con mayores niveles de degradación.

\title{
Palabras clave
}

Bosques andinos, cámaras de seguimiento, degradación de hábitat, polinización planta-ave.

\section{ESTADO DE CONSERVACIÓN DEL ZAMBULLIDOR PLATEADO NORTEÑO PODICEPS JUNINENSIS}

\author{
Esteban A. Guevara', Tatiana Santander ${ }^{1, *}$, Adrián Soria ${ }^{1}$, Pierre-Yves Henry ${ }^{2}$ \\ ${ }^{1}$ Aves y Conservación-BirdLife en Ecuador, Pasaje Joaquín Tinajero E3-05 y Jorge Drom, Casilla 17-17 906, \\ Quito, Ecuador.
}

2Muséum National d’Histoire Naturelle, Département Écologie et Gestion de la Biodiversité, París, Francia.

*Autora para correspondencia; E-mail: tsantander@avesconservacion.org

Los zambullidores son aves acuáticas que pertenecen al orden Podicipediformes, uno de los grupos más sensibles a los cambios que ocurren en sus hábitats, ya que son altamente dependientes de los humedales. En el presente trabajo se presenta una revisión respecto de cambios recientes en la distribución y tendencias poblacionales de la subpoblación del norte del Zambullidor Plateado Norteño Podiceps juninensis. El Zambullidor Plateado Norteño está catalogado como Vulnerable en Ecuador y En Peligro en Colombia, mientras que a nivel global se considera Casi Amenazada. Se ha considerado información histórica y reciente proveniente de varias fuentes, así como la obtenida a través de los Censos Neotropicales de Aves Acuáticas realizados desde 2004 en varios humedales del Ecuador. Este análisis nos ha permitido conocer que el Zambullidor Plateado Norteño ha sufrido extirpaciones locales y procesos de colonización en las últimas tres décadas. Una importante reducción de la población ha ocurrido en las lagunas de la provincia de Imbabura. Existen registros recientes, aunque en bajo número, en lagunas de Tungurahua y Chimborazo. Actualmente, más del $90 \%$ de la subpoblación se reparte en dos lagunas: La Mica y Colta. Esta última está siendo severamente afectada por la colmatación y reducción del espejo de agua, poniendo en peligro el futuro del humedal y de la especie. En cuanto a la tendencia de la población, se ha mantenido estable en La Mica (entre 2004 y 2013), mientras que en Colta exhibe un crecimiento acelerado. Se considera que la colonización en Colta se realizó por aves provenientes de La Mica, cuando se construyó el embalse que afectó a la vegetación acuática de este humedal. La ausencia de reportes recientes en humedales de Colombia sugiere que la especie permanece en números muy bajos, lo cual resalta la contracción que ha sufrido la porción más norteña de la distribución de la especie y su prioridad de conservar los sitios donde actualmente habita.

Palabras clave

Población, amenazadas, conservación, Colta, Micacocha, Podiceps juninensis. 


\title{
DIVERSIDAD Y DISTRIBUCIÓN DE AVIFAUNA EN 2020 HECTÁREAS DE BOSQUE Y MATORRAL SECO TROPICAL EN DAULAR, GUAYAS
}

\author{
Nancy Hilgert*, Virgilio Benavides, George Varela, Paul Abad, Francisco Hernández-Baquero \\ BENHIL Cía. Ltda., Guayaquil, Ecuador. \\ *Autora para correspondencia; E-mail: benavideshilgert@gmail.com
}

Presentamos resultados de 624 horas de observaciones directas e indirectas realizadas entre 10 de noviembre y 30 diciembre de 2014 en 2020 hectáreas de bosque y matorral seco tropical con árboles de guayacán, capuey, bototillo, ceibo, pechiche, ébano, sapote de perro y varias especies más de flora, en Daular, provincia de Guayas. Recorrimos diez transectos de $1000 \mathrm{~m}$ de longitud cada uno. Registramos 106 especies de aves pertenecientes a 92 géneros, 40 familias y 18 órdenes. Entre los registros relevantes constan siete especies endémicas tumbesinas y amenazadas de extinción: Amazona lilacina (EN), Brotogeris pyrrhoptera (EN), Crypturellus transfasciatus (NT), Psittacara erythrogenys (NT), Ortalis erythroptera (VU), Leptotila ochraceiventris (VU) y Spinus siemiradzkii (VU). Las restantes 99 especies se categorizan como Preocupación Menor (LC); 77 de estas son residentes y 2 son migratorias boreales: Falco peregrinus y $F$. columbarius. En base a la clasificación de gremios alimenticios se registraron 30 especies insectívoras, 29 carnívoras, 21 omnívoras, 17 granívoras, 5 frugívoras y 4 nectarívoras. Las especies más abundantes fueron Dives warczewiczi, Euscarthmus meloryphus, Cathartes aura y Cyanocorax mystacalis. La diversidad alfa definida mediante los índices de Simpson D, Shannon y Pieliu indicó que el sur del predio (1000 ha de bosque y matorral seco) es más diverso que el sector norte, lo que puede deberse a la variada vegetación presente en este sector. La diversidad beta mostró que la riqueza es "aceptable" con un valor del 77\%; 66 especies son compartidas entre el norte y sur del predio.

Palabras clave

Avifauna, distribución de poblaciones, diversidad, endemismo, bosque seco, Guayas.

\section{MALARIA Y OTROS PARÁSITOS HEMOSPORIDIOS EN LAS AVES DE GALÁPAGOS}

\section{Maricruz Jaramillo*, Patricia G. Parker}

Department of Biology, University of Missouri-St. Louis, One University Blvd. St. Louis, MO 63121, Estados Unidos.

*Autora para correspondencia; E-mail: mari.jaramillo@umsl.edu

Las poblaciones de fauna isleña se consideran más vulnerables a patógenos introducidos por su aislamiento de agentes de enfermedades. En Galápagos, estudios de enfermedades realizados por nuestro grupo han revelado la existencia de varios patógenos en la avifauna endémica de las islas, entre los cuales se han encontrado dos géneros de hemoparásitos: Plasmodium y Haemoproteus. Hemos identificado cuatro linajes de Plasmodium, comúnmente denominado malaria aviar, de los cuales uno aparece repetidamente en diferentes especies de aves, localidades y años de muestreo. Sin embargo, no hemos detectado evidencia de que este parásito pueda completar su ciclo de vida en las aves de Galápagos. Continuos hallazgos del parásito sugieren que en Galápagos debe estar presente un reservorio natural. Por tanto, investigamos el rol que las aves introducidas en Galápagos puedan tener en el establecimiento y la transmisión de enfermedades parasitarias. También esperamos revelar la taza de exposición al parásito por medio de ensayos por inmunoadsorción ligado a enzimas que detecten la presencia de anticuerpos contra Plasmodium. Mientras tanto, un parasito Haemoproteus del subgénero Haemoproteus (H. multipigmentatus) es altamente prevalente en las Palomas de Galápagos Zenaida galapagoensis. A pesar de que este subgénero es específico para los Columbiformes, lo hemos encontrado en varias aves paseriformes en la isla Santiago. Nuevamente, las paseriformes no parecen ser hospederos competentes, pero estos eventos de "derramamiento" del parásito (spillover) pueden ser los precursores a un cambio de hospedero. Por medio de nuestra investigación esperamos aumentar el conocimiento que se tiene sobre la ecología de enfermedades que afectan a ecosistemas aislados y determinar si poblaciones de aves de este grupo de islas, a $1000 \mathrm{~km}$ de la costa ecuatoriana, son más susceptibles a enfermedades.

Palabras clave

Enfermedades de aves, Galápagos, Haemoproteus, malaria aviar, Plasmodium. 


\title{
PRIMER REGISTRO DE PARÁSITOS SUBCUTÁNEOS DEL GÉNERO PHILORNIS (DIPTERA: MUSCIDAE) EN PICHONES DEL MATORRALERO CABECIPÁLIDO ATLAPETES PALLIDICEPS (AVES: EMBERIZIDAE)
}

\author{
Mery Elizabeth Juiña ${ }^{1,2}$ \\ 'Fundación de Conservación Jocotoco, Lizardo García E9-104 y Andrés Xaura, Quito, Ecuador. \\ -Museo Ecuatoriano de Ciencias Naturales del Instituto Nacional de Biodiversidad, Rumipamba 341 y Av. De \\ los Shyris, Quito, Ecuador. \\ E-mail: meryj_bio@yahoo.com
}

Los parásitos de las aves silvestres en Ecuador han sido poco estudiados. Sin embargo, se conoce que estos vertebrados pueden ser afectados por ácaros, pulgas, garrapatas, piojos y moscas, problema que se agrava cuando la especie receptora es particularmente vulnerable. El Matorralero Cabecipálido Atlapetes pallidiceps es un ave endémica del Ecuador, considerada en peligro de extinción. Sus principales problemas son el parasitismo reproductivo del Vaquero Brilloso Molothrus bonariensis y la pérdida de hábitat. Actualmente se han detectado parásitos subcutáneos en los pichones del Matorralero Cabecipálido y el efecto de su presencia todavía es desconocido. Con el fin de determinar el tipo de parásito subcutáneo e investigar su ciclo de vida, se procedió a monitorear los nidos infectados mediante observaciones directas durante la temporada reproductiva, entre los años 2003 y 2006 (febrero-mayo). El 54\% de los nidos registrados tuvo éxito reproductivo y de estos, el $28 \%$ estuvo infectado con parásitos subcutáneos del género Philornis. Este fenómeno no ha causado mortalidad ni deformación en sus pichones. El género Philornis juegan un papel importante en poblaciones pequeñas de aves como los pinzones de Darwin, causando una drástica disminución poblacional. La determinación de la especie de parásito es muy importante a fin de prevenir algún tipo de impacto negativo en el Matorralero Cabecipálido.

Palabras clave

Atlapetes pallidiceps, época reproductiva, parásitos subcutáneos, Philornis.

\section{AVES ENDÉMICAS TUMBESINAS EN LA ZONA DE VISITANTES DE CERRO BLANCO EN GUAYAQUIL (2013-2015)}

\author{
Javier López*, Aleyda Quinteros, Jenny Castro \\ Universidad de Guayaquil, Víctor Emilio Estrada y Costanera, Guayaquil, Ecuador. \\ *Autor para correspondencia; E-mail: javier.lopezr@ug.edu.ec
}

La finalidad de esta investigación fue constatar la diversidad de aves que se pueden identificar en los senderos de la zona de visitantes del Bosque Protector Cerro Blanco, con énfasis en las especies endémicas tumbesinas, por su potencial interés para los observadores de aves y su aplicación en el aviturismo. La investigación es cualitativa y bibliográfica, basada en investigaciones previas y guías de identificación de campo de donde obtuvimos información de rangos de distribución, ecología y características diagnósticas. Además, realizamos observaciones de campo y empleamos fichas de observación. En casos puntuales, solicitamos el criterio de un experto para valorar la identificación de campo. Realizamos 45 sesiones de observación entre abril de 2013 y abril de 2015. El número de especies identificadas fue similar entre sesiones de observación, según esfuerzo y recorrido. Algunas especies endémicas tumbesinas fueron frecuentes, de fácil observación e identificación. La frecuencia de avistamientos de las especies endémicas tumbesinas fue variable; desde un 2,5\% en especies infrecuentes (p.ej. Campephilus gayaquilensis, Myiopagis subplacens), a un $82 \%$ en especies comunes, fáciles de identificar y regulares en la fauna urbana (p.ej. Furnarius cinnamomeus, Campylorhynchus fasciatus). Un grupo de aves se ubicó entre el 48-64\% de los registros. Este grupo incluye aves de particulares características, que se pueden emplear en la promoción, comunicación e incentivo a la actividad de observación (p.ej. Arremon abeillei, Cyanocorax mystacalis, Veniliornis callonotus).

\section{Palabras clave}

Aviturismo, diversidad, endemismo, Bosque Protector Cerro Blanco, Guayas. 


\title{
DISTRIBUCIÓN POTENCIAL DEL PERICO PECHIBLANCO PYRRHURA ALBIPECTUS: FACTORES CLIMÁTICOS QUE CONDICIONAN SU PRESENCIA
}

\author{
Christian Mendoza', Katiusca Valarezo ${ }^{1, *}$, Marlon Cobos ${ }^{1,2}$ \\ 'Zoológico Municipal de Loja, Ecuador. \\ 2 Facultad de Biología, Universidad de La Habana, Cuba. \\ *Autora para correspondencia; E-mail: katuvalarezo@hotmail.com
}

El Perico Pechiblanco Pyrrhura albipectus es un perico amenazado, endémico del sureste de Ecuador y el norte de Perú. En base a sus registros se considera que su área de distribución (cría) es de alrededor de $6300 \mathrm{~km}^{2}$. No obstante, nuevas localidades sugieren que su área de ocupación podría incrementarse. Hasta hoy no se han realizado análisis de los factores climáticos que condicionan su distribución, ni de la configuración espacial de la misma. Nuestro objetivo fue identificar la distribución potencial del Perico Pechiblanco y los factores climáticos que condicionan su presencia. Para esto, desarrollamos un modelo de nicho ecológico correlativo de la especie, utilizando registros de presencia y variables bioclimáticas. Filtramos los datos de presencia para disminuir la auto-correlación espacial. Seleccionamos las variables por su valor de contribución y evitamos aquellas con mayor colinearidad $(<0,8)$. Con estos datos generamos un modelo en el programa Maxent con 50 réplicas. Los principales factores que condicionaron la distribución potencial de la especie fueron el rango anual de temperatura, la estacionalidad de la temperatura y la precipitación del mes más húmedo. Las mayores probabilidades de presencia de esta especie se localizaron en las estribaciones orientales de los Andes y en la provincia de Zamora Chinchipe. Altas probabilidades de presencia se extendieron a las zonas centro y oriental de Perú, y a la Costa de Ecuador. Las áreas de presencia potencial separadas por barreras biogeográficas se consideraron como sobre-predicciones. Otros sectores climáticamente adecuados, alejados de la distribución conocida de la especie, aunque conectados con ésta, podrían albergar equivalentes ecológicos de esta especie o mantener interacciones no favorables para ella. Esto explicaría la ausencia de la especie en gran parte del área de presencia potencial encontrada. Futuros esfuerzos son necesarios para identificar posibles nuevas poblaciones de esta especie, las limitantes de su distribución y con ello su real estado de conservación.

\section{Palabras clave}

Adecuación de hábitat, endemismo, modelado de nicho ecológico, Psittacidae.

\section{DIVERSIDAD DE AVES EN ÁREAS VERDES DE LA CIUDAD DE QUITO, ECUADOR}

\section{Eliana Montenegro-Pazmiño*, Diego F. Cisneros-Heredia}

Universidad San Francisco de Quito, Colegio de Ciencias Biológicas y Ambientales, Laboratorio de Zoología Terrestre, Calle Diego de Robles y Vía Interoceánica, campus Cumbayá, Edif. Darwin, of. DW-010A, Casilla Postal 17-1200-841, Quito, Ecuador.

*Autora para correspondencia; E-mail: elimontenegrop@yahoo.com

La avifauna de Quito ha disminuido con el pasar de los años y la creciente urbanización. Chapman (1926) registró 102 especies en lo que hoy constituye el núcleo urbano de Quito; las estimaciones más actuales corresponden aproximadamente a 40 especies. El presente estudio corresponde al primer acercamiento a nivel ecológico de la diversidad de aves en Quito, por medio del estudio de sus áreas verdes. Se realizaron dos metodologías de colección de datos: transectos y puntos de observación. Registramos 65 especies en 16 áreas verdes dentro del núcleo urbano de Quito. Zenaida auriculata, Zonotrichia capensis y Turdus fuscater fueron las especies más comunes de la ciudad. Presentan alimentación omnívora y granívora, y se encuentran en hábitats no muy complejos de la ciudad, aprovechando el medio antrópico. Las especies poco comunes y raras fueron en su mayoría insectívoras y frugívoras, con preferencia por hábitats más complejos, encontrándose en mayor densidad en parques y áreas verdes que contienen parches de vegetación nativa y quebradas. Se encontró una correlación negativa entre impacto humano y riqueza en las áreas verdes. Determinar las cifras actuales de aves en Quito, y analizar su entorno, es importante para incentivar un mejor manejo de la ciudad a nivel ecológico, promoviendo así la conservación y recuperación de la avifauna nativa.

\section{Palabras clave}

Avifauna, ecología urbana, frecuencia, riqueza, Quito. 


\title{
FUNDACIÓN JOCOTOCO: 18 AÑOS CONSERVANDO LAS AVES AMENAZADAS EN ECUADOR
}

\author{
Eliana Montenegro-Pazmiño*, Martin Schaefer \\ Fundación de Conservación Jocotoco, Lizardo García E9-106 y Andrés Xaura, P.O. Box 17-16-337, Quito, \\ Ecuador. \\ *Autora para correspondencia; E-mail: e.montenegro@fjocotoco.org
}

Ecuador es un paraíso de aves; con 1588 especies, se ubica en el quinto lugar de países con mayor diversidad de aves. Las 82 familias existentes en el país corresponden al 91\% de las familias presentes en la región. Existen 36 especies endémicas distribuidas en importantes biorregiones de alto endemismo y diversidad, como son las bajuras del Chocó, las bajuras tumbesinas, estribaciones orientales y occidentales de los Andes y las bajuras amazónicas. En el país se encuentran 99 especies globalmente amenazadas según la UICN. De estas, 8 están Críticamente Amenazadas (CR), 23 están En Peligro (EN) y 68 son Vulnerables (VU). La Fundación Jocotoco se constituyó en 1998 con el principal objetivo de conservar el hábitat de la Gralaria Jocotoco Grallaria ridgelyi, descubierta por Robert Ridgely en un viaje al cerro Tapichalaca, Zamora Chinchipe. Así se fundó la primera reserva de la fundación, que actualmente cuenta con 10 reservas en todo el país, creadas con el mismo objetivo que Tapichalaca: "proteger especies globalmente amenazadas en el Ecuador continental". Todas las reservas de la Fundación Jocotoco se encuentran dentro de distintas IBA y EBA, tal es el caso del Zamarrito Pechinegro Eriocnemis nigrivestis en la Reserva Yanacocha, al noroccidente de Quito; el Matorralero Cabecipálido Atlapetes pallidiceps, en la Reserva Yunguilla, al sur del Azuay; el Perico de Orcés Pyrrhura orcesi, en la Reserva Buenaventura, al sur de la provincia de El Oro; y la Estrellita Esmeraldeña Chaetocercus berlepschi, en la Reserva Ayampe, provincia de Manabí. La Fundación Jocotoco realiza constantes monitoreos de estas especies, así como acciones directas de conservación que han llevado al incremento de sus poblaciones dentro de sus reservas.

\section{Palabras clave}

Aves amenazadas, conservación, Fundación Jocotoco, reservas.

\section{INFLUENCIA DE LA GANADERÍA SOBRE LA COMUNIDAD DE AVES DE PÁRAMO EN EL PARQUE NACIONAL CAJAS}

\author{
Andrea V. Nieto1*, Gabriela M. Samaniego', Pedro X. Astudillo', Steven Latta ${ }^{2}$, Catherine Graham², \\ Boris A. Tinoco ${ }^{1}$ \\ 'Laboratorio de Ecología, Escuela de Biología, Ecología y Gestión, Universidad del Azuay, Av. 24 de Mayo \\ 7-77 y Hernán Malo, Cuenca, Ecuador. \\ ${ }^{2}$ National Aviary (USA). Allegheny Commons West, Pittsburgh, Estados Unidos. \\ 3Department of Ecology and Evolution, Stony Brook University, 650 Life Sciences Building, Stony Brook, \\ NY11794, Estados Unidos. \\ *Autora para correspondencia; E-mail: andreanieto87@gmail.com
}

La ganadería en los páramos representa una gran amenaza para este ecosistema y su biodiversidad, afecta la estructura del ecosistema y provoca alteraciones como la disminución en la disponibilidad de recursos, pérdida de hábitat y homogenización del paisaje. Las aves son un grupo especialmente sensible a los cambios de hábitat y podrían verse afectadas por estas alteraciones. En el Parque Nacional Cajas (Andes sur del Ecuador), el ganado ha sido removido en su mayoría; sin embargo, en algunas zonas este disturbio aún persiste. Investigamos la influencia de la ganadería sobre la riqueza, abundancia y diversidad funcional de aves de páramo del Parque Nacional Cajas. Para el estudio establecimos dos tratamientos: alta y baja presencia de ganado. Las aves fueron registradas a través de transectos de $1 \mathrm{~km}$ de longitud, con siete transectos en cada tratamiento. Además, consideramos variables ambientales como altura y cobertura del pajonal, cantidad de fecas de ganado y heterogeneidad espacial. A través de modelos lineales mixtos generalizados (GLMM) exploramos la influencia de la ganadería y variables ambientales sobre la comunidad 
de aves. Los sitios con ganadería presentaron mayor número de fecas, mientras que los sitios sin ganadería presentaron mayor cobertura y altura del pajonal. La heterogeneidad espacial fue levemente mayor en sitios sin ganadería, a pesar de no haber encontrado diferencias significativas. La ganadería no mostró influencia sobre la riqueza y abundancia. Sin embargo, en términos de diversidad funcional, los modelos indicaron que tanto la dispersión funcional y riqueza funcional, están asociados positivamente con sitios con baja ganadería y son más heterogéneos. Se conoce que la ganadería ejerce un efecto sobre la comunidad de aves a través de la modificación del hábitat. Nuestros resultados sugieren que esta transformación podría también afectar la función de ciertas especies que dependen de estructuras vegetales complejas para mantener sus roles ecológicos.

\title{
Palabras clave
}

Alteración, aves, diversidad funcional, ganadería, Parque Nacional Cajas.

\section{UN CUENTO SOBRE DOS AVES TERRESTRES: NEOMORPHUS RADIOLOSUSY ARAMIDES WOLFI}

\author{
Jorge Olivo ${ }^{1}$, Luke Browne ${ }^{1,2}$, Scott Walter ${ }^{1,2}$, Jordan Karubian, ${ }^{1,2,}$ \\ ${ }^{1}$ Fundación para la Conservación de los Andes Tropicales (FCAT), Mariano Hurtado N 50-89 y Vicente \\ Heredia, Quito, Ecuador. \\ ${ }^{2}$ Department of Ecology \& Evolutionary Biology, Tulane University, 400 Boggs Building, New Orleans, LA, \\ 70118, Estados Unidos. \\ *Autor para correspondencia; E-mail: jk@tulane.edu
}

Las aves terrestres pueden ser muy susceptibles a las perturbaciones causadas por el ser humano como la pérdida y fragmentación de hábitat y la cacería. En el noroccidente de Ecuador estudiamos dos especies de aves terrestres que están consideradas En Peligro de extinción por UICN y son poco conocidas: el Cuco Hormiguero Franjeado Neomorphus radiolosus y el Rascón Montes Moreno Aramides wolfi. En este trabajo presentamos los resultados de estudios de comportamiento de nidificación, movimientos y los efectos de la fragmentación de bosque sobre estas dos especies en la Reserva Ecológica Mache Chindul. Nuestros estudios mostraron que A. wolfi prefiere anidar en bosque secundario, mientras que $N$. radiolosus se encuentra en bosque primario. También, $A$. wolfi tiene un rango de movimiento más pequeño que $N$. radiolosus en bosque continuo. Entre los 22 fragmentos de bosque que muestreamos, $A$. wolfi (observado en 14 fragmentos) es mucho más común que $N$. radiolosus (no observado en fragmentos). Sugerimos que los factores del paisaje (como elevación y cobertura de bosque alrededor del fragmento) y factores locales (como el área del fragmento y la calidad de hábitat dentro del fragmento) afectan la probabilidad de observar $A$. wolfi, y que las diferencias de biología básica pueden explicar el hecho de que el uno sea mucho más común que el otro.

\section{Palabras clave}

Aramides wolfi, aves terrestres, conservación, Chocó, Esmeraldas, fragmentos, Neomorphus radiolosus, Reserva Ecológica Mache Chindul.

\section{ANÁLISIS DE LA DIVERSIDAD DE AVES DEL ARCHIPIÉLAGO DE JAMBELÍ, EL ORO, ECUADOR}

\author{
Adrian Orihuela-Torres*, Fausto López, Leonardo Ordóñez-Delgado \\ Departamento de Ciencias Biológicas, Universidad Técnica Particular de Loja, San Cayetano Alto, Calle \\ París, Loja, Ecuador. \\ *Autor para correspondencia; E-mail: aaorihuela@utpl.edu.ec
}

Estudiamos la diversidad y abundancia de aves del archipiélago de Jambelí, provincia de El Oro, suroccidente de Ecuador. Este archipiélago posee una extensión de 41700 ha, de las cuales 15000 corresponden a manglar. El archipiélago de Jambelí es considerado como un Área de Importancia para la Conservación de Aves. A pesar de su importancia, la composición de las comunidades de aves en el área no ha sido estudiada de forma adecuada. El presente trabajo se efectuó entre agosto 2015 y julio 2016. Desactions censos por medio de 
puntos de conteo, transectos y recorridos en bote alrededor de cada isla poblada: Costa Rica, Bellavista, Pongalillo, Las Huacas y Las Casitas. Utilizamos el método de rarefacción para analizar la riqueza de especies por localidad de estudio, siendo la isla Las Casitas la que más diversidad presentó (60 especies). El índice de Jaccard, que establece el grado de similitud entre las poblaciones de aves de las diferentes islas, dio resultados superiores al 50\% de similitud en todos los casos, llegando hasta el $76 \%$ entre las islas Las Huacas y Pongalillo. En total registramos 106 especies de aves pertenecientes a 41 familias. Del total de especies, 7 son endémicas de la región Tumbesina, 29 son migratorias boreales, 4 son migratorias australes y una es migratoria intratropical. Además, registramos tres especies consideradas amenazadas en Ecuador: Aramides axillaris (Vulnerable), Falco peregrinus (Vulnerable) y Rallus longirostris (En Peligro). De manera adicional, recabamos información detallada del primer registro documentado de reproducción de Gampsonyx swainsonii en Ecuador, e iniciamos el seguimiento de la dieta de una colonia de Athene cunicularia. Este trabajo constituye el primer esfuerzo de estudio sistemático de las comunidades de aves de este archipiélago.

\section{Palabras clave}

Archipiélago de Jambelí, comunidad de aves, conservación, diversidad.

\section{LA “GIGA DIVERSIDAD” DE AVIFAUNA DEL SUR DEL ECUADOR}

\section{Renzo Paladines Puertas ${ }^{1, *}$, Leonardo Ordóñez-Delgado², Trotsky Riera Vite', Matt Clark ${ }^{1}$, Fabián Rodas López ${ }^{1}$}

${ }^{1}$ Naturaleza \& Cultura Internacional, Av. Pío Jaramillo A. y Venezuela, Loja, Ecuador. 2Departamento de Ciencias Biológicas, Universidad Técnica Particular de Loja, San Cayetano Alto, Calle París, Loja, Ecuador.

*Autor para correspondencia; E-mail: rpaladip@naturalezaycultura.org

A pesar de su reducida superficie, Ecuador se encuentra entre los 17 países mega-diversos del mundo, siendo el cuarto país en diversidad de aves con 1679 especies enlistadas. Los números son más sorprendentes a través del análisis de metadatos y nuevas observaciones de aves en 33 sitios del sur del Ecuador. Entre las provincias de El Oro, Loja y Zamora Chinchipe hemos determinado la presencia de 995 especies de aves (60\% del total nacional) en una superficie equivalente a solo el 11\% del país $\left(27491,80 \mathrm{~km}^{2}\right)$. Sin duda, estos números, sumados a los de las plantas (60\% del total de vasculares) y mamíferos (35\% del total nacional), demuestran una muy alta concentración de especies por unidad de superficie. Por ello, sugerimos que el sur del Ecuador es una de las áreas "giga-diversas" a nivel mundial. Varias son las causas que se analizan: la influencia de la depresión de Huancabamba y la topografía compleja del sur de Ecuador, patrones históricos de glaciación, clima y refugios pleistocénicos. Estos factores en conjunto dan lugar a la presencia en nuestra región de estudio de siete de las diez Áreas de Endemismo de Aves registradas en Ecuador. Asimismo, existen siete ecosistemas (océano, manglar, matorral seco interandino, matorral húmedo interandino, bosque nublado, páramo y bosque lluvioso amazónico) en apenas $236 \mathrm{~km}$ longitudinales de distancia. Finalmente, analizamos aspectos socio económicos, estado actual de los ecosistemas, esfuerzos de conservación que se han implementado -incluyendo medidas del Estado, privadas y comunitarias- y su relación con las 26 Áreas de Importancia para la Conservación de las Aves declaradas en estas tres provincias.

\section{Palabras clave}

Conservación, diversidad, El Oro, endemismo, Loja, Zamora Chinchipe. 


\title{
Paolo Piedrahita ${ }^{1}$, Hermann Wagner ${ }^{2}$
}

${ }^{1}$ Facultad de Ciencias de la Vida, ESPOL, Guayaquil, Ecuador. 2Institut für Biologie II, RWTH Aachen, D-52074 Aachen, Alemania.

*Autor para correspondencia; E-mail: ppiedra@espol.edu.ec

La Lechuza de Campanario Tyto alba punctatissima es una de las dos especies de Strigiformes que habitan las islas Galápagos. En este archipiélago, la cadena trófica del ecosistema terrestre se conforma por un número limitado de especies. Por lo tanto, se esperaría que el rol depredador de las lechuzas esté estrechamente relacionado a la abundancia de sus presas. En el presente estudio presentamos datos preliminares de la dieta y comportamiento de forrajeo de la Lechuza de Campanario de las Galápagos dentro de la zona agrícola de la isla Santa Cruz. La presencia de lechuzas se registró en 25 sitios a lo largo de esta zona. Los registros se confirmaron mediante la observación de egagrópilas, plumas e individuos presentes en nidos o dormideros. Recolectamos un total de 103 egagrópilas para el análisis de sus contenidos, cuyos datos preliminares muestran que la dieta se compone principalmente de roedores, ortópteros y rara vez de aves. Adicionalmente, dos lechuzas fueron equipadas con transmisores tipo GiPSy No. 5, de Technosmart, por dos noches seguidas. Los datos de los transmisores sugieren que las lechuzas forrajearon en el mismo sitio en ambas noches, a una distancia aproximada de $500 \mathrm{~m}$ desde el dormidero, sin abandonar la zona agrícola de la isla. Además, los datos también mostraron que ambas lechuzas volaron durante la noche de forma independiente, utilizando varios sitios de percha y/o descanso. El monitoreo de dos parejas adicionales, cuyos dormideros se ubicaron también alrededor de la pareja equipada con trasmisores, sugiere que la distancia territorial entre parejas reproductivas es de aproximadamente $500 \mathrm{~m}$. Los datos preliminares del presente estudio brindan una primera percepción del comportamiento y estrategias de caza de las lechuzas de campanario de las Galápagos.

Palabras clave

Dieta, Galápagos, Lechuza de Campanario, uso de hábitat.

\section{PREFERENCIA ALIMENTARIA DEL ZAMARRITO PECHINEGRO ERIOCNEMIS NIGRIVESTIS EN LA LADERA NOROCCIDENTAL DEL VOLCÁN PICHINCHA}

\author{
Cristian Poveda ${ }^{1,2, *}$, Bryan Rojas ${ }^{1,2}$, Tatiana Santander ${ }^{1}$, Esteban A. Guevara ${ }^{1}$ \\ ${ }^{1}$ Aves y Conservación - BirdLife en Ecuador, Pasaje Joaquín Tinajero E3-05 y Jorge Drom, Casilla 17-17 \\ 906, Quito, Ecuador \\ ${ }^{2}$ Escuela de Ciencias Biológicas, Pontificia Universidad Católica del Ecuador, Av. 12 de Octubre 1076 y \\ Roca, Quito, Ecuador. \\ *Autor para correspondencia; E-mail:cristian.poveda@hotmail.com
}

El Zamarrito Pechinegro Eriocnemis nigrivestis es un colibrí endémico de Ecuador, restringido a dos localidades: ladera noroccidental de volcán Pichincha y cordillera del Toisán, en Imbabura. La especie se considera Críticamente Amenazada debido a su distribución reducida $\left(<70 \mathrm{~km}^{2}\right)$ y su reducido tamaño poblacional (<1000 individuos). Poco se conoce acerca de la ecología alimenticia de la especie, aunque se ha reportado que puede alimentarse de hasta 29 especies de plantas. Sin embargo, la intensidad con la que la especie explota diferentes recursos florales se desconoce. Realizamos un estudio sobre preferencia alimenticia en la población del Pichincha entre septiembre 2014 y abril 2016. Examinamos un total de 22 especies de plantas en las reservas Yanacocha, Verdecocha y Cerro La Esperanza. Utilizamos cámaras de seguimiento colocadas frente a las flores, y programas para detección de movimiento. Obtuvimos 1400 horas de video en las cuales registramos 141 visitas de E. nigrivestis a siete de 22 plantas estudiadas. Las especies Palicourea fuchsioides (Rubiaceae) y Macleaenia rupestris (Ericaceae) fueron las más visitadas. La primera es una especie amenazada y endémica de Ecuador que produce flores casi todo el año, excepto durante julio y agosto (época seca). En nuestros sitios de estudio, esta planta crece solamente en la reserva Verdecocha, donde E. nigrivestris ha sido observado consistentemente durante los últimos años. Recomendamos investigar el grado de especialización tanto del Zamarrito Pachinegro como de Palicourea fuchsioides para evaluar la importancia 
de la planta como recurso alimenticio. Igualmente, recomendamos un monitoreo continuo de las interacciones planta-colibrí, con el fin de entender el efecto de la fenología floral sobre las preferencias alimentarias del Zamarrito Pechinegro. Por su bajo costo y facilidad de implementación, el uso de cámaras trampa y herramientas de detección de movimiento posibilita extender este tipo de estudios a la poco conocida población de Toisán.

Palabras clave

Cámaras de seguimiento, dieta, Macleania rupestris, Palicourea fuchsioides, Zamarrito Pechinegro.

\section{PRIMERAS OBSERVACIONES DE LA DIETA DEL BÚHO OREJICORTO ASIO FLAMMEUS BOGOTENSIS (STRIGIDAE) EN PICHINCHA, ECUADOR}

\section{Glenda M. Pozo-Zamora1,*, Jorge Brito', Rubí García1, Ibeth Alarcón², Héctor Cadena-Ortiz ${ }^{1,3}$}

${ }^{1}$ Museo Ecuatoriano de Ciencias Naturales del Instituto Nacional de Biodiversidad, División de Ornitología y Mastozoología, Calle Rumipamba 341 y Av. de los Shyris, Quito, Ecuador.

${ }_{2}^{2}$ Área de Investigación y Monitoreo de Avifauna, Aves y Conservación-BirdLife en Ecuador, Joaquín Tinajero E3-05 y Jorge Drom, Quito, Ecuador.

${ }^{3}$ Programa de Reparación Ambiental y Social (PRAS), Ministerio del Ambiente, Av. La Coruña E25-58 y San Ignacio, Quito, Ecuador.

*Autora para correspondencia; E-mail: glenda.pozo@yahoo.es

Damos a conocer las primeras observaciones de la dieta del Búho Orejicorto Asio flammeus bogotensis en tres localidades de la provincia de Pichincha, Ecuador. A partir de 52 egagrópilas, determinamos el consumo de 167 presas de 22 especies. La dieta estuvo compuesta mayoritariamente por mamíferos pequeños $(50,8 \%)$, seguidos por insectos $(43,1 \%)$, aves $(4,7 \%)$, anfibios $(0,5 \%)$ y reptiles $(0,5 \%)$. El ítem que aportó mayor biomasa fueron las crías de Sylvilagus brasiliensis, con un 36\%. El índice de Shannon indicó una mediana diversidad de presas consumidas. Encontramos que la dieta de A. flammeus bogotensis en Pichincha está formada principalmente por roedores, similar a otros estudios de la misma especie en Norte y Sudamérica. No hallamos relación entre la masa corporal de las presas y su frecuencia en las egagrópilas, lo que nos sugiere que el Búho Orejicorto no está depredando selectivamente por el tamaño de presas sino por su disponibilidad.

\section{Palabras clave}

Andes, Asio flammeus bogotensis, dieta, egagrópilas, Pichincha.

\section{DIETA DE FALCO SPAR VERIUS (FALCONIFORMES: FALCONIDAE) EN DOS TIPOS DE HÁBITATS INTERANDINOS, NORTE DE ECUADOR}

\section{Glenda M. Pozo-Zamora*, Jonathan Aguirre, Jorge Brito M.}

Museo Ecuatoriano de Ciencias Naturales del Instituto Nacional de Biodiversidad, División de Ornitología, Entomología y Mastozoología, Calle Rumipamba 341 y Av. de los Shyris, Quito, Ecuador.

*Autora para correspondencia; E-mail: glenda.pozo@yahoo.es

El Cernícalo Americano Falco sparverius es una rapaz diurna ampliamente distribuida desde Alaska hasta Tierra de Fuego. Su dieta generalista está basada principalmente en invertebrados. En Ecuador existe escasa información biológica de esta especie. En el presente trabajo damos a conocer la dieta del Cernícalo Americano por medio del análisis de 201 egagrópilas colectadas en dos hábitats en la región interandina del norte de Ecuador. Los ítems más representativos en Sangolquí y Tababela fueron los coleópteros (48,4 y $39,5 \%$, respectivamente), seguidos por ortópteros (31,3 y 30,7\%, respectivamente). En términos de biomasa, los mamíferos fueron los más importantes dentro de la dieta del Cernícalo Americano, con el 40,3\% en Sangolquí y el 70,8\% en Tababela. El índice de Shannon $\left(H^{\prime}=1,894\right)$ indicó una mediana diversidad de presas. La amplitud de dieta $(0,26)$ indicó que esta especie tuvo una dieta especialista, que podría ser una respuesta funcional a la disponibilidad de presas, distinta de otros hábitats donde presenta dieta generalista. 
Encontramos que los ítems alimenticios no presentan una diferencia significativa entre los dos sitios de estudio.

\title{
Palabras clave
}

Falco sparverius, alimentación, dieta, egagrópilas, presas, Andes.

\section{PRIMERA EVIDENCIA DEL COMPORTAMIENTO DE DORMIR DURANTE EL VUELO: ESTUDIO DE MOVIMIENTO Y NEUROFISIOLOGÍA EN FRAGATAS (FREGATA MINOR) EN GALÁPAGOS}

\author{
Niels C. Rattenborg1,*, Bryson Voirin ${ }^{1,2}$, Sebastian M. Cruz ${ }^{3}$, Ryan Tisdale', Giacomo Dell'Omo ${ }^{4}$, Hans- \\ Peter Lipp5, Martin Wikelski ${ }^{3,6}$, Alexei L. Vyssotski ${ }^{7}$ \\ ${ }^{1}$ Avian Sleep Group, Max Planck Institute for Ornithology, Seewiesen, 82319, Alemania. \\ ${ }^{2}$ California Academy of Sciences, San Francisco, California, 94118, Estados Unidos. \\ ${ }^{3}$ Department of Migration and Immuno-Ecology, Max Planck Institute for Ornithology, Radolfzell, 78315, \\ Alemania. \\ ${ }^{4}$ Ornis Italica, Rome, 00199, Italia. \\ ${ }^{5}$ Institute of Anatomy, University of Zurich, CH-8053, Zurich, Suiza. \\ ${ }^{6}$ Department of Biology, University of Konstanz, 78457 Konstanz, Alemania. \\ ${ }^{7}$ Institute of Neuroinformatics, University of Zurich and Swiss Federal Institute of Technology (ETH), \\ Zurich, 8057, Suiza. \\ *Autor para correspondencia; E-mail: rattenborg@orn.mpg.de
}

Varias especies de aves pueden mantener vuelos sostenidos durante días o semanas. Esto demanda una explicación de si las aves duermen durante el vuelo y cómo es esto posible. Dado que los delfines pueden nadar mientras duermen con un solo hemisferio cerebral y un ojo cerrado a la vez, se piensa comúnmente que las aves también poseen esta capacidad, Pero debido a la ausencia de registros de la actividad cerebral, se desconoce con certeza si esto es factible en aves. Mediante el registro del electroencefalograma (EEG) de individuos de Fragata Grande Fregata minor volando sobre el océano durante 10 días, demostramos que duermen de modo uni-hemisférico y bi-hemisférico durante el vuelo, pero solo en pequeñas cantidades. Por la noche, y solo mientras planean, la actividad EEG de ondas lentas (AOL; 0,75 a 4,5 Hz de potencia) - un indicador de la intensidad de dormir de ondas lentas (DOL)- aumentó por periodos que duran hasta varios minutos. Aunque dormir es más asimétrico durante el vuelo que en tierra, $28 \%$ fue bi-hemisférico, demostrando que para el control aerodinámico no es necesario dormir con un hemisferio a la vez. En su lugar, una relación entre la dirección del vuelo y la asimetría inter-hemisferios en la AOL y gamma $(30-80 \mathrm{~Hz})$, la cual está relacionada con la atención visual, sugieren que las fragatas utilizan el DOL uni-hemisferio para ver hacia dónde van durante el sueño. La atención proporcionada por dormir con un solo hemisferio cerebral es aparentemente insuficiente para satisfacer todas las exigencias ecológicas, por lo que las fragatas dormían solo 1,4 horas/día en vuelo, $<11 \%$ de la cantidad en tierra. Se cree que las fragatas, otra aves y mamíferos marinos concilian la paradoja de tener que dormir durante largos periodos de actividad mediante el sueño uni-hemisférico. En cambio, podrían renunciar a grandes cantidades de dormir cuando las circunstancias ecológicas también requieren toda su atención.

Palabras clave

Dormir en vuelo, fisiología, Fregata minor, Galápagos. 


\title{
CONOCIMIENTO POPULAR Y CULTURAL SOBRE LOS BÚHOS EN LA CIUDAD DE LOJA, ECUADOR
}

\author{
Santiago Rivera-Romero, ${ }^{1, *}$, Juan Manuel García ${ }^{2}$, Juan Freile³, Leonardo Ordóñez-Delgado² \\ 'Universidad Técnica Particular de Loja, Loja, Ecuador. \\ ${ }^{2}$ Sección de Manejo y Gestión de Recursos Naturales, Departamento de Ciencias Biológicas, Universidad \\ Técnica Particular de Loja, Loja, Ecuador. \\ ${ }^{3}$ Comité Ecuatoriano de Registros Ornitológicos. \\ *Autor para correspondencia; E-mail: sarivera2@utpl.edu.ec
}

Las principales amenazas que enfrentan las poblaciones de búhos en la región tropical están relacionadas con el uso de plaguicidas, accidentes en carreteras, tráfico ilegal, trasformación del paisaje, cacería ilegal y destrucción de hábitats. Además, han sido objeto de actitudes de miedo, lo cual ha generado mitos, leyendas, creencias o supersticiones, como sucede también con arañas, serpientes, murciélagos y grandes depredadores. Suele considerarse a los búhos como aves de mal agüero. Esto se ha documentado en varios lugares del mundo, entre ellos Costa Rica, África tropical y Colombia. En el presente estudio pretendemos determinar el grado de conocimiento y las actitudes de la población de la ciudad de Loja respecto a las especies de búhos, mediante el uso de encuestas. Realizamos 360 encuestas dirigidas a adultos y jóvenes, de dos diferentes generaciones y de ambos géneros. En general, la mayoría de las actitudes y percepciones sobre estas aves fueron positivas, ya que un $84,80 \%$ de la población encuestada mencionó tener interés por conservarlas y un $80,24 \%$ consideró que son importantes para el medio ambiente. Por el contrario, un 21,84\% los considera de mal agüero y un $14,34 \%$ los temen o los consideran peligrosos. Encontramos diferencias en el nivel de conocimiento entre jóvenes y adultos. Personas de mayor edad mostraron mayor conocimiento sobre búhos.

Palabras clave

Actitudes, búhos, etnozoología, Loja, percepciones, Strigidae.

\section{MODELAMIENTO DE NICHO ECOLÓGICO DEL GUACAMAYO VERDE MAYOR ARA AMBIGUUS GUA YAQUILENSIS: IMPLICACIONES PARA SU CONSERVACIÓN}

\author{
Carlos Fabián Viteri Herrera*, Carola Bohórquez Litardo \\ Fundación Bioeducar, Gral. Córdova y Mendiburu, Guayaquil, Ecuador. \\ *Autor para correspondencia; E-mail: carfviteri@gmail.com
}

Se estima que la población de la subespecie Ara ambiguus guayaquilensis está declinando rápidamente debido a la fragmentación y deforestación de los bosques que habita, expansión de la frontera urbana y agrícola, y tráfico ilegal. El objetivo de esta investigación fue desarrollar modelos predictivos de distribución potencial del Guacamayo Verde Mayor en la cordillera Chongón Colonche, para desarrollar lineamientos preliminares de conservación de la especie. Recopilamos 57 registros georreferenciados de la especie a partir de bases de datos de libre acceso como eBird y GBIF, así como de estudios de análisis de vacíos, estudios de muestreo de poblaciones en Chongón Colonche y de información de la Dirección de Medio Ambiente del Gobierno Provincial del Guayas. La distribución potencial y actual de la especie se modeló mediante el método de Máxima Entropía con el software Maxent 3.3.3. Seleccionamos ocho variables bioclimáticas de las 19 variables de Worldclim, en base a las variables que aportaron la mayor contribución al modelo mediante el test de Jacknife. Para la evaluación de los modelos aplicamos el análisis del área bajo la curva (Curva ROC). Realizamos un análisis de omisiones de conservación de la especie, comparando la distribución modelada de la especie y límites geográficos de sitios bajo algún régimen de conservación. Además, generamos una propuesta preliminar de sitios prioritarios para la declaratoria de áreas de conservación y para reintroducción de la especie en base a álgebra de mapas, que pueden fortalecer los esfuerzos de conservación estatal, privada o comunitaria.

\section{Palabras clave}

Álgebra de mapas, áreas de conservación, especies amenazadas, Maxent, modelos de nicho ecológico, reintroducción, Ara ambiguus guayaquilensis. 


\title{
LA COBERTURA DE BOSQUE ALREDEDOR DE FRAGMENTOS ES UN DETERMINANTE DE LA DIVERSIDAD DE AVES FRUGÍVORAS EN LOS FRAGMENTOS
}

\section{Scott T. Walter ${ }^{1,2}$, Jorge Olivo ${ }^{1}$, Luke Browne ${ }^{1,2}$, Jordan Karubian ${ }^{1,2, *}$}

'Fundación para la Conservación de los Andes Tropicales (FCAT), Mariano Hurtado N 50-89 y Vicente Heredia, Quito, Ecuador.

${ }_{2}^{2}$ Department of Ecology \& Evolutionary Biology, Tulane University, 400 Boggs Building, New Orleans, LA, 70118, Estados Unidos.

*Autor para correspondencia; E-mail: jordankarubian@gmail.com

Las aves que comen frutos son importantes para la regeneración y mantenimiento de los bosques tropicales, entre otros motivos, por la dispersión de semillas que éstas realizan. El papel de las aves frugívoras de gran tamaño corporal es especialmente importante, ya que solo ellas pueden comer y dispersar semillas grandes tales como palmas y aguacates. La pérdida y fragmentación de los bosques tropicales puede tener un fuerte impacto en estas especies y su mutualismo con las plantas. Para entender la situación actual, estudiamos la importancia de factores locales (interior de fragmentos, hábitats) versus factores del paisaje (elevación y cobertura forestal alrededor de fragmentos) para determinar la diversidad del gremio de aves frugívoras grandes. Muestreamos 22 fragmentos de bosque a través de transectos de $500 \mathrm{~m}$ para especies de tucanes, cotingas, pavas y chachalacas. Además, también incluimos psitácidos, ya que estos pueden dispersar semillas viables. Muchas de estas especies se encuentran en peligro según la UICN. Nuestro resultado más relevante establece que los factores del paisaje son más importantes que los factores locales dentro los fragmentos. La cobertura de bosque alrededor de cada fragmento influyó significativamente en la diversidad y probabilidad de encuentro de nuestras especies focales. La matriz que rodea un fragmento incrementa la oportunidad de forrajeo y también el movimiento entre fragmentos, si dentro de estos se presenta un cierto nivel de cobertura de árboles. Esperamos que estos resultados incentiven a propietarios de terrenos para mantener algunos árboles al momento de desbrozar los predios, o permitir el crecimiento de árboles en áreas de agricultura o ganadería.

\section{Palabras clave}

Aves frugívoras, cobertura de bosque, conservación, especies amenazadas, fragmentos, Reserva Ecológica Mache Chindul.

\section{PRESENTACIONES EN PÓSTERS}

\section{DIVERSIDAD DE AVIFAUNA DEL ÁREA NACIONAL DE RECREACIÓN PARQUE LAGO}

\author{
Evelyng Astudillo-Sánchez ${ }^{1}$, Elisa López¹, Jimmy Astudillo', George Varela², Nancy Hilgert de \\ Benavides $^{2}$ \\ 1Universidad Espíritu Santo, Guayaquil, Ecuador. \\ ${ }^{2}$ BenHil Cía. Ltda., Guayaquil, Ecuador. \\ *Autora para correspondencia, E-mail: eveavesecuador@gmail.com
}

El Área Nacional de Recreación Parque Lago (ANRPL) fue establecida como parte del Sistema Nacional de Áreas Protegidas del Ecuador en 2003. Está ubicada en el cantón Guayaquil, km 25-30 en la vía a la costa. Es un embalse que desde 1992 represa las aguas de los ríos Chongón y Perdido, con una superficie aproximada de 2000 ha, y comprende principalmente el espejo de agua y una franja terrestre alrededor, de $50 \mathrm{~m}$ de ancho, que mantiene remanentes de bosque seco y áreas intervenidas para uso agrícola y pecuario. El objetivo del presente estudio fue aportar información para la actualización del Plan de Manejo del ANRPL realizado en 2007, caracterizando la avifauna del área protegida asociada al humedal artificial, mediante la revisión de datos recogidos durante 12 meses en los años 2010 y 2011. El método aplicado para el registro de aves fue el de puntos de conteo. Se registró en dos puntos diferentes a todas las aves vistas y escuchadas dentro de un 
radio de $50 \mathrm{~m}$ durante $30 \mathrm{~min}$. También se incluyeron aves identificadas fuera del radio mencionado. Se registró un total de 81 especies de aves durante los 12 meses, siendo el Cormorán Neotropical Phalacrocorax brasilianus la especie más abundante por un amplio margen $(55,81 \%)$. El número de individuos registrados fue mayor en septiembre de 2010 y septiembre de 2011. El valor del índice de Shannon-Wiener fue de 1,96 y el valor del índice de Simpson fue de 0,67. Estos valores nos indican la riqueza del área protegida y su importancia para la avifauna y biodiversidad regional.

Palabras clave

Abundancia, aves, diversidad, humedal artificial, riqueza, Shannon-Wiener, Simpson.

\title{
DIVERSIDAD AVIFAUNÍSTICA DEL SECTOR DOS MANGAS DEL BOSQUE PROTECTOR CHONGÓN COLONCHE, SANTA ELENA
}

\author{
Evelyng Astudillo-Sánchez ${ }^{1,3, *}$, Camila Tamayo', Jimmy Astudillo1, Enzo R. Reyes ${ }^{1,2}$ \\ 'Universidad Espíritu Santo, Guayaquil, Ecuador. \\ ${ }_{2}^{2}$ Museo de Ballenas, Salinas, Ecuador. \\ ${ }^{3}$ Universidad Nacional Mayor de San Marcos, Lima, Perú. \\ *Autora para correspondencia, E-mail: eveavesecuador@gmail.com
}

El presente estudio forma parte del proyecto de investigación "Los colibríes como estrategia para la conservación de los bosques secos y de garúa presentes en la cordillera Chongón Colonche". La cordillera Chongón Colonche tiene una superficie de $95 \mathrm{~km}$ en sentido este-oeste y está ubicada dentro de la región de endemismo Tumbesina; 44000 ha de esta cordillera están protegidas como bosque protector (BP). El BP Chongón Colonche es un Área de Importancia para la Conservación de las Aves (IBA EC025), donde se han registrado 171 especies. El sector Dos Mangas, dentro del BP Chongón Colonche, ha sido poco explorado en relación a su avifauna. Por ello, el presente estudio se orientó a caracterizar la avifauna de Dos Mangas. Empleamos transectos lineales de $1 \mathrm{~km}$, combinados con cinco puntos de conteo, mismos que fueron muestreados entre mayo y agosto de 2015. Se registraron 1939 individuos pertenecientes a 145 especies, que equivale al 84,79\% del total de especies registradas en el BP. Las 145 especies pertenecen a 37 familias. De estas especies, 24 son endémicas tumbesinas, 2 son endémicas de las bajuras del Chocó, 1 de la Sierra del suroeste; 1 de las bajuras tumbesinas y 1 es endémica de Ecuador. Asimismo, de las 145 especies encontradas dos están catalogadas como En Peligro (EN), 5 son Vulnerables (VU) y 1 Casi Amenazada (NT). El área alberga una diversidad de avifauna media-alta. La diversidad es bastante uniforme y similar a la registrada con la Reserva Ecológica Comunal Loma Alta. Debido al potencial del área, recomendamos que se implemente un programa de monitoreo para conservación de los recursos mediante patrones de diversidad presente en los principales tipos de vegetación.

Palabras clave

AICA, Bosque Protector Chongón Colonche, diversidad, endemismo.

\section{FACTORES DE HÁBITAT QUE INFLUENCIAN LA ABUNDANCIA Y EL NICHO ECOLÓGICO DEL CÓNDOR ANDINO EN LOS ANDES SUR DE ECUADOR}

\author{
Pedro X. Astudillo ${ }^{1}$, David C. Siddons ${ }^{1}$, Edwin Zárate', Santiago Barros $Q^{1}$, Juan A. Orellana ${ }^{1 * *}$, Steven \\ C. Latta ${ }^{2}$ \\ 'Universidad del Azuay, Escuela de Biología, Ecología y Gestión, Av. 24 de Mayo 7-77, Cuenca, Ecuador. \\ ${ }^{2}$ National Aviary (USA), Allegheny Commons West, Pittsburgh, Estados Unidos. \\ *Autor para correspondencia; E-mail: juanoj1@ hotmail.com
}

La población del Cóndor Andino Vultur gryphus se encuentra en declinación. En Ecuador, la mayoría de esfuerzos de monitoreo se han concentrado en el norte del país. Estos monitoreos no han considerado los factores de hábitat (altitud, pendiente, asociaciones vegetales, clima) que influencian la abundancia y el nicho 
ecológico de la especie. Para investigar esto, se realizaron visitas de campo mediante transectos, puntos de conteo y búsquedas de registros históricos en bases de datos especializadas para los Andes sur del Ecuador. Las visitas de campo fueron seleccionadas a partir de la generación de un modelo de distribución (método Maxent). En total se registraron 39 individuos, 13 en las visitas de campo y 26 en los registros históricos. La abundancia de cóndores incrementa significativamente con la altitud, mientras que el páramo influenciado por ganado cimarrón no explica el número cóndores. Los resultados señalan que las localidades de mayor altitud son los sitios más convenientes; sin embargo, en adición a la altitud, la pendiente del terreno presenta una ligera tendencia a explicar positivamente la abundancia de cóndores. Así, al modelar el nicho ecológico del Cóndor Andino (método ENFA y MADIFA), los resultados para el 48,3\% del área de estudio señalan que la pendiente y páramo contienen la mayor variación (variación del primer eje: $43,4 \%$ ) del nicho de la especie, mientras que la menor variación (variación del segundo eje: 33,8\%), que define el nicho más ajustado (especialidad), está asociada a la elevación e intervención del hábitat. Consecuentemente, cambios pequeños en la condición del páramo (intervención de hábitat) y en la elevación del terreno, sugieren cambios mayores en la dimensión del nicho ecológico del Cóndor Andino. El presente estudio pretende contribuir con información para realizar actividades de conservación en la región de los Andes Sur del Ecuador.

\title{
Palabras clave
}

Altitud, Cóndor Andino, estructura del hábitat, modelo de distribución, páramo.

\section{DIVERSIDAD DE AVES EN FRAGMENTOS DE BOSQUE EN EL CHOCÓ ECUATORIANO}

\section{Luke Browne ${ }^{1,2}$, Fernando Castillo' ${ }^{2}$ Juan Rivero' ${ }^{2}$, Andrea Moreno ${ }^{2}$, Luis Carrasco ${ }^{2}$, Scott Walter ${ }^{1,2}$, Renata Ribeiro, ${ }^{1,2}$, Jordan Karubian ${ }^{1,2, *}$}

'Department of Ecology \& Evolutionary Biology, Tulane University, 400 Boggs Building, New Orleans, LA, 70118, Estados Unidos.

${ }^{2}$ Fundación para la Conservación de los Andes Tropicales (FCAT), Mariano Hurtado N 50-89 y Vicente Heredia, Quito, Ecuador.

*Autor para correspondencia; E-mail: jordankarubian@gmail.com

La pérdida y fragmentación de hábitats es muy común en Ecuador como en otros países megadiversos, pero su impacto en las aves varía mucho. Para entender mejor la causa de esta variación realizamos un estudio para identificar los factores que determinan la diversidad de aves del sotobosque en fragmentos de bosque. Atrapamos aves con redes de neblina en casi 40 fragmentos de bosque en la Reserva Ecológica Mache Chindul, noroccidente de Ecuador. Analizamos cómo los factores del paisaje (elevación y cobertura de bosque alrededor del fragmento) y factores locales (área del fragmento y calidad de hábitat dentro del fragmento) afectan la riqueza, diversidad y composición de la comunidad de aves del sotobosque. También analizamos cómo los patrones de diversidad de aves varían en el mismo fragmento con el transcurso del tiempo, para identificar posibles cambios poblacionales. Encontramos que los factores del paisaje son importantes en la determinación de la diversidad de aves, dependiendo del gremio, y que la diversidad varía mucho con el transcurso del tiempo. Discutimos nuestros resultados en relación a la conservación de la diversidad en paisajes fragmentados.

\section{Palabras clave}

Composición de comunidades, diversidad, fragmentos, redes de neblina, Reserva Ecológica Mache Chindul.

\section{LA RESERVA AVES DEL PARAÍSO, CERRO ESCALERA, NORTE DEL PERÚ}

\author{
Gisella Carabelli \\ BirdingWomen, Playa Ayampe, Manabí, Ecuador. \\ Autor para correspondencia; E-mail: gcarabelli@hotmail.com
}

La reserva Aves del Paraíso se encuentra en la zona de amortiguamiento de la reserva Cerro Escalera, norte de Perú. En este momento, la reserva tiene una superficie aproximada de 500 ha, con un rango altitudinal de 500 
2000 m s.n.m. Parte de esta área son fincas compradas con fondos de nuestra empresa de aviturismo BirdingWomen. La otra parte fue entregada en concesión por la reserva Cerro Escalera. El proyecto nació con el propósito de terminar con la depredación ambiental en las fincas aledañas a la reserva Cerro Escalera. Los objetivos principales del proyecto son: 1) brindar apoyo a la conservación de los bosques lluviosos y de neblina de la zona; 2) regresar estos bosques a su estado natural; y 3) compartir este lugar con todos aquellos que quieren estudiar el ecosistema muy biodiverso, especialmente la avifauna. Este lugar único, por varias razones geográficas permaneció inaccesible por muchos años debido al conflicto político en el Perú. Por ello, son pocos los estudios que se han realizado en el sector. BirdingWomen quiere brindar la oportunidad a las personas interesadas en realizar investigaciones en el sitio. Con este afán, estamos construyendo una cabaña y una plataforma de observación de aves, y alistamos un sitio de acampar para que los investigadores y voluntarios puedan permanecer en la reserva y ayudar a establecer un registro de todas las especies. Phaethornis koepckeae es una de las especies endémicas presente en Aves del Paraíso. La reserva está ubicada a solo 40 min de Tarapoto, en la carretera a Yurimaguas, $\mathrm{km} 32$. El acceso desde Ecuador es muy fácil y cruza por algunos de los territorios más importantes de avistamiento de aves: Loja, Podocarpus, Tapichalaca, Balsas, Jaén, Huembo, Alto Mayo, Moyobamba y Tarapoto.

Palabras clave

Conservación, Reserva Aves del Paraíso, Tarapoto, aviturismo.

\section{LA MONJITA TRICOLOR DE MORFO CANELO LONCHUIRA MALACCA EN ECUADOR}

\section{Orlando Carrión ${ }^{1 *}$, Carola Bohórquez ${ }^{1}$, Olaf Jahn², Juan José Álava ${ }^{3}$}

'Fundación Ecológica Andrade, Cerro Masvale, Km 16, vía Boliche-Puerto Inca, Unidad Educativa Naranjal, Ecuador.

2Zoological Research Museum A. Koenig (ZFMK), Leibniz Institute for Animal Biodiversity, Adenauerallee 160, 53113 Bonn, Alemania.

${ }^{3}$ Fundación Ecuatoriana para el Estudio de Mamíferos Marinos (FEMM), Guayaquil, Ecuador.

*Autor para correspondencia; E-mail: fundacionandrade@yahoo.com

Las monjitas del género Lonchura pertenecen a la familia Estrildidae. La Monjita Tricolor L. malacca fue introducida en Ecuador alrededor del año 1998. Hasta el momento, ha sido identificada solamente en base a imágenes fotográficas publicadas en el segundo reporte anual del Comité Ecuatoriano de Registros Ornitológicos. Originalmente se identificó como Monjita Castaña L. atricapilla, la cual posee muchos rasgos morfológicos y de comportamiento similares al morfo canelo de la Monjita Tricolor L. malacca. La diferenciación taxonómica entre estas dos especies es un proceso complejo y presenta desafíos de investigación desde el punto de vista de la diferenciación de coloración, morfología externa, comparación de cantos y la aplicación de métodos genéticos. Con el fin de dilucidar la correcta identificación de la especie presente en Ecuador, seguimos realizando estudios de campo en el cruce de Milagro-Yaguachi y en la parroquia Taura, sector La Lagartera, provincia del Guayas. Aquí presentamos las primeras grabaciones de vocalizaciones. Comparamos las llamadas nasales de monjitas grabadas en Guayas con ejemplos disponibles en Xeno-Canto (http://www.xeno-canto.org) de especies del género Lonchura, pero no conseguimos resultados totalmente concluyentes. Basados en la comparación preliminar de llamadas nasales, la especie se ha identificado como L. malacca, aunque existen por lo menos dos grabaciones de L. atricapilla jagori en Xeno-Canto que contienen llamadas nasales muy similares a las de L. malacca. Además, destacamos que las poblaciones de monjitas observadas en Ecuador no poseen dimorfismo sexual lo que representa otro indicio para que la identificación como L. malacca se considere correcta. Durante las grabaciones de cantos observamos varias veces a un individuo llevando residuos de flores de caña de azúcar Saccharum officinarum en su pico, indicando un comportamiento de anidación en el cruce de Milagro. Recomendamos continuar el monitoreo de esta especie para investigar su historia natural y establecer un plan de manejo para su control en el Ecuador.

\section{Palabras clave}

Especie introducida, Guayas, identificación, Lonchura atricapilla, Lonchura malacca, morfo castaño. 


\title{
REVISIÓN BIBLIOGRÁFICA DE FACTORES AMBIENTALES QUE INFLUYEN LA DINÁMICA POBLACIONAL DEL PINGÜINO DE GALÁPAGOS
}

\author{
Andrea Coloma Villacrés ${ }^{1, *}$, Paolo Piedrahita Piedrahita', Gustavo Jiménez Uzcáteguii ${ }^{2}$ \\ ${ }^{1}$ Escuela Superior Politécnica del Litoral, Guayaquil, Ecuador. \\ ${ }^{2}$ Fundación Charles Darwin, Galápagos, Ecuador. \\ *Autora para correspondencia; E-mail: adcoloma@espol.edu.ec
}

La respuesta ecológica a eventos naturales como El Niño se refleja en la población de Pingüinos de Galápagos Spheniscus mendiculus. Entre los años 1965 y 2004 se registraron nueve fenómenos de El Niño, de los cuales dos fueron categorizados como fuertes y siete como débiles. Esto sugiere que el aumento de los eventos de El Niño debilita la recuperación poblacional de pingüinos, haciéndolos vulnerables al cambio climático. Tras varios años de estudio se ha podido identificar que el aumento de la frecuencia de eventos El Niño, que es una de las consecuencias del cambio climático, no permitirá que la población se recupere. Con el objetivo de priorizar investigaciones futuras, el presente trabajo comprende una revisión de las investigaciones realizadas hasta la fecha sobre los factores abióticos y bióticos que influyen en la dinámica poblacional del Pingüino de Galápagos. Para comprender la variabilidad de la dinámica poblacional, a partir del año 2010 se mantiene un censo poblacional anual mediante captura y recaptura. Estos censos poblacionales permiten inferir sobre la supervivencia, mortalidad y ciclos reproductivos. Otra preocupación es la interacción de especies introducidas como los gatos, quienes son transmisores de enfermedades como la toxoplasmosis. Además, la prevalencia del parásito Plasmodium sp., que provoca la malaria aviar. Todos estos factores pueden dar como resultado una disminución en la población de pingüinos. Sin embargo, según los censos poblacionales anuales, la población del Pingüino de Galápagos tiene una tendencia estable o incluso a aumentar. Futuros trabajos deberán considerar el impacto de las enfermedades en las subpoblaciones de pingüinos, lo cual también influyen en la dinámica poblacional.

Palabras clave

Cambio climático, dinámica poblacional, El Niño, factores ambientales, Spheniscus mendiculus.

\section{AHORRO ENERGÉTICO NOCTURNO EN CUATRO ESPECIES DE COLIBRÍES EN LOS ANDES DEL AUSTRO ECUATORIANO}

\author{
Gabriela Córdova ${ }^{1}$, Gabriela Urgilés ${ }^{1 *}$, Anusha Shankar² \\ 1Universidad del Azuay, Cuenca, Ecuador. \\ ${ }^{2}$ Stony Brook University, New York, Estados Unidos. \\ *Autora para correspondencia; E-mail: gabrielaurgiles1@ outlook.com
}

Con el cambio climático, la temperatura ambiente se ha incrementado, presentando un desafío en organismos heterotermos que dependen del ahorro de energía por las noches. Estos cambios en la temperatura los están forzando a disminuir su tasa metabólica y ahorrar energía. Se han realizado varios estudios sobre torpor, pero en condiciones de laboratorio. El objetivo de este estudio es conocer cuál es la estrategia de los colibríes con diferente rol de comportamiento para manejar su energía en un hábitat de clima variable. Especialmente, cómo usan el torpor en las noches para equilibrar su presupuesto energético. Procuraremos capturar cinco individuos de cuatro especies de colibríes que hayan cumplido sus actividades en su hábitat natural. Mediante este estudio se puede predecir que los comportamientos de las especies alterarán el número y la duración de los periodos de torpor, considerando que la temperatura ambiental es apta para que los colibríes entren en estado de torpor. Predecimos que los colibríes generalistas (Metallura tyrianthina) y ruteros (Coeligena iris) utilizarán más torpor que los colibríes territoriales (Heliangelus viola, Aglaeactis cupripennis).

\section{Palabras clave}

Trochilidae, comportamiento, temperatura, torpor. 


\title{
EL PERICO DE EL ORO Y SU ROL EN LA CONSERVACIÓN DEL PATRIMONIO NATURAL PROVINCIAL DE EL ORO
}

\section{César Garzón-Santomaro*, Mario H. Yánez-Muñoz}

Museo Ecuatoriano de Ciencias Naturales del Instituto Nacional de Biodiversidad (MECN-INB), Rumipamba 341 y Av. de los Shyris, Quito, Ecuador.

*Autor para correspondencia; E-mail: cesar.garzon@ambiente.gob.ec

El Perico de El Oro Pyrrhura orcesi es una especie endémica de Ecuador, En Peligro de extinción, restringida a los bosques nublados de las provincias de El Oro y Azuay, en el suroccidente del país. Su distribución no incluye el Sistema Nacional de Áreas Protegidas, y prácticamente se restringe a la reserva Buenaventura, manejada por la Fundación de Conservación Jocotoco. Desde 2002, varios estudios se han enfocado en monitorear su dinámica y genética poblacional, e identificar nuevas poblaciones en su área distribución. Con esta información generada por los investigadores del MECN-INB, se concibió la estrategia de conservación de esta ave endémica, que vincula y fomenta la participación activa de los gobiernos locales y regionales para proteger las poblaciones de la especie y sus hábitats, los cuales concentran gran diversidad de flora y fauna única de los bosques nublados de Ecuador. La estrategia se enfoca en el diseño y creación del Corredor Ecológico "Pahua-Buenaventura-Guayacán" para la protección de 350 especies de aves, más de 100 especies de anfibios y reptiles, y especies de mamíferos y flora que al momento son desconocidas. El diseño de este corredor integra a los gobiernos parroquiales, municipales y el Gobierno Autónomo Descentralizado Provincial de El Oro, para promover los valores culturales de responsabilidad ambiental y el empoderamiento de la conservación del patrimonio natural de la provincia. Nuestra estrategia también busca que el corredor ecológico suscite el establecimiento de acuerdos recíprocos para el uso de los sistemas hídricos de las microcuencas que conforman parte del corredor. Actualmente, la implementación de la primera fase de este proyecto ha definido el estado actual de los ecosistemas ayudando a tomar decisiones que motiven la conservación de su biodiversidad y de su especie emblemática de ave: el Perico de El Oro.

\section{Palabras clave}

Conservación, corredor ecológico Pahua-Buenaventura-Guayacán, El Oro, monitoreo, Pyrrhura orcesi.

\section{DIVERSIDAD DE AVES NOCTURNAS EN FRAGMENTOS DE BOSQUE EN EL CHOCÓ ECUATORIANO}

\author{
Nelson González², Luke Browne1,2, Scott T. Walter ${ }^{1,2}$, Julio Loor², Michael Darkes², Juan Freile², \\ Thomas Gillespie ${ }^{4}$, Jordan Karubian 1,2*
}

'Department of Ecology \& Evolutionary Biology, Tulane University, 400 Boggs Building, New Orleans, LA, 70118, Estados Unidos.

${ }^{2}$ Fundación para la Conservación de los Andes Tropicales (FCAT), Mariano Hurtado N 50-89 y Vicente Heredia, Quito, Ecuador.

${ }^{3}$ Comité Ecuatoriano de Registros Ornitológicos.

*Autor para correspondencia; E-mail: jordankarubian@gmail.com

Las aves nocturnas son depredadores importantes en muchos ecosistemas, pero sus patrones de diversidad asociados con distintos hábitats, y el impacto de la conversión de hábitats en este grupo faunístico ha sido difícil de documentar, especialmente en bosques tropicales en los cuales las aves nocturnas son poco conocidas. Evaluamos el impacto de la fragmentación de bosque en una comunidad de aves nocturnas por medio de 264 periodos de muestreo en 22 fragmentos de bosque en la Reserva Ecológica Mache Chindul, noroeste de Ecuador. Analizamos el efecto de variables de hábitat (altura de dosel, apertura del dosel y densidad de árboles grandes) y variables del fragmento (área, elevación y cobertura de bosque alrededor del fragmento) respecto de la riqueza de especies, composición de la comunidad y probabilidad de detección de estas aves. Documentamos 11 especies de aves nocturnas en total, con un promedio de 3,4 $\pm 1,4$ ( \pm SD; rango $=2-7)$ por fragmento, con más especies en fragmentos más grandes, más bajos en elevación y con 
doseles más abiertos. También descubrimos que las tasas de detección eran más altas durante muestreos más largos y en la madrugada (versus la puesta del sol). Estas relaciones con características de hábitat, el fragmento y formas de muestreo avanzan nuestro entendimiento de la ecología y conservación de aves nocturnas, un gremio escasamente estudiado en bosques neotropicales.

Palabras clave

Aves nocturnas, diversidad, elevación, fragmentos, Reserva Ecológica Mache Chindul.

\title{
CONTEO POBLACIONAL DEL CANCLÓN ANHIMA CORNUTA DURANTE LA ESTACIÓN HÚMEDA EN LA LAGUNA CANCLÓN, RESERVA ECOLÓGICA MANGLARES CHURUTE
}

\author{
George Agustín Gutiérrez Borbor ${ }^{1,2, *}$, Mireya Pozo Cajas², Paolo Piedrahita Piedrahita ${ }^{2}$ \\ 'Reserva Ecológica Manglares Churute, Km. 49 de la vía Guayaquil-Machala, sector Churute, Ecuador. \\ 2Escuela Superior Politécnica del Litoral, Campus Gustavo Galindo Velásquez Km 30,5 vía Perimetral, \\ Guayaquil, Ecuador. \\ *Autor para correspondencia; E-mail: george16george@ @otmail.com
}

Estudiamos la población del canclón Anhima cornuta para definir su estado de conservación en la laguna Canclón (Reserva Ecológica Manglares Churute), en la costa del Ecuador. El estudio se efectuó desde mediados de marzo hasta finales de mayo 2015, durante la estación húmeda. Con el fin de conocer la densidad poblacional del canclón, se realizaron censos sobre $8 \mathrm{~km}$ alrededor del perímetro de la laguna, en donde se definieron ocho puntos de observación que presentaban una buena cobertura de avistamientos de esta especie. Adicionalmente, los puntos escogidos difirieron en cuanto a rangos de perturbación (presencia de granjas, ganadería y agricultura). La abundancia de canclones disminuyó en los puntos más perturbados, con porcentajes de observación entre 5-22\% (11-48 individuos). Esta especie depende de la vegetación de los humedales, la cual es proporcional a la abundancia de canclones (mayor cobertura vegetal, mayor abundancia). Con la recopilación de estos datos se analizó el estado de la población de A. cornuta,comparando con estudios anteriores. Encontramos una posible disminución en las densidades por puntos de observación en relación a las zonas de mayor perturbación. Estas zonas ponen en peligro tanto el hábitat del canclón como la supervivencia del humedal, que se reconoce como sitio Ramsar.

Palabras clave

Anhima cornuta, especie amenazada, humedales, población, Ramsar.

\section{INTENTOS DE REPRODUCCIÓN DE LA GAVIOTA REIDORA LEUCOPHAEUS ATRICILLA EN LAS PISCINAS DE ECUASAL (SANTA ELENA): ¿UNA NUEVA ESPECIE DE GAVIOTA QUE SE REPRODUCE EN ECUADOR?}

\author{
Ben Haase1,*, Enzo M. R. Reyes ${ }^{1,2}$ \\ 'Museo de Ballenas, Av. General Enríquez Gallo 11-09, Salinas, Ecuador. \\ ${ }^{2}$ Colectivo "Seas and Forests", Santa Elena, Ecuador. \\ *Autor para correspondencia; E-mail: bhaase2012@gmail.com
}

Durante más de 25 años, personal del Museo de Ballenas, en Salinas, realiza el monitoreo sistemático de las aves acuáticas en las piscinas de Ecuasal, donde la Gaviota Reidora Leucophaeus atricilla es un migratorio boreal común, con presencia durante todo el año. Desde 2008 se han registrado diferentes individuos de Gaviota Reidora y un individuo presumiblemente híbrido entre esta especie y la Gaviota Cabecigrís Chroicocephalus cirrocephalus en comportamientos de cortejo y defensa de territorio. Los intentos de establecimiento de nidada comenzaron en 2014 a través de típicas posturas de defensa de territorio en las piscinas de Ecuasal, en Pacoa. En el mismo año se confirmó la primera nidada de esta especie en el país, a través de observaciones de aves incubando. Para evitar perturbaciones, no hubo verificación. Observaciones consecutivas a través del periodo reproductivo mostraron la falla de cría. El lugar donde se descubrió la nidada corresponde al mismo lugar donde se observó por primera 
vez a la pareja en actividad de cortejo en 2008, por lo cual sugerimos que se trata de los mismos individuos. Durante el mismo año, se observó una pareja mixta compuesta por el supuesto híbrido y otro individuo de Gaviota Cabecigrís también en fase de incubación, pero tampoco tuvieron éxito. Durante el censo mensual de mayo de 2016 se registró nuevamente a un individuo de Gaviota Reidora "puro" con una pareja de Gaviota Cabecigrís incubando. El tamaño de la nidada fue de dos huevos, ligeramente más alargados y más oscuros que los de Gaviota Cabecigrís, que poco tiempo después fueron abandonados por su infertilidad.

Palabras claves

Anidación, Chroicocephalus cirrocephalus, híbridos, Leucophaeus atricilla, reproducción, Ecuasal, Santa Elena.

\title{
COSTOS DE TERMORREGULACIÓN EN CUATRO ESPECIES DE COLIBRÍES EN LOS ANDES SUR DEL ECUADOR
}

\author{
Ana C. Morales'*, Anusha Shankar1, Catherine H. Graham1, Donald R. Powers ${ }^{2}$ \\ 'Stony Brook University, Department of Ecology and Evolution, New York, Estados Unidos. \\ ${ }^{2}$ George Fox University, Department of Biology, Newberg, Estados Unidos. \\ *Autora para correspondencia; E-mail: anacmorales9@gmail.com
}

Los colibríes son pequeños endotermos que presentan altas tasas metabólicas, lo cual les impide acumular grasa fácilmente. Debido a esto, necesitan manejar su presupuesto energético adecuadamente para sobrevivir. Alrededor del $30 \%$ de este presupuesto es utilizado en termorregulación. Si existieran cambios en la temperatura ambiente (p.e., cambio climático), los colibríes tendrían que modificar su presupuesto energético para mantener su temperatura corporal constante. El objetivo de este estudio es determinar cómo la variación de temperatura puede afectar los costos diarios de termorregulación de cuatro especies de colibríes (Metallura thyriantina, Aglaeactis cupripennis, Coeligena iris y Heliangelus viola) en los Andes del sur del Ecuador. El trabajo se realizó en la Estación Científica El Gullán (2800-3000 m s.n.m.), un sitio que presenta temperaturas muy variables a lo largo del día $\left(0-40^{\circ} \mathrm{C}\right)$. Para realizar este estudio, medimos la tasa metabólica de cada especie bajo varios rangos de temperatura controlada. Así, esperamos construir un modelo de costos diarios de termorregulación y probar cómo estos pueden cambiar bajo diferentes temperaturas registradas en el campo. Este modelo será aplicado en otros sitios con diferentes regímenes de temperatura en donde se encuentran las mismas especies. Se conoce que la temperatura termoneutral de los colibríes es alrededor de $\operatorname{los} 32-37^{\circ} \mathrm{C}$. Es por esto que esperamos que los costos de termorregulación sean más altos en sitios con temperaturas más bajas y más variables.

Palabras clave

Andes, cambio climático, tasa metabólica, temperatura, termorregulación, Trochilidae.

\section{LAS AVES DEL BOSQUE PROTECTOR LA PROSPERINA, GUAYAQUIL, ECUADOR}

\author{
Mireya Pozo Cajas*, Aleyda Quinteros Trelles \\ Escuela Superior Politécnica del Litoral, Campus Gustavo Galindo Velasco Km 30,5 vía Perimetral, \\ Guayaquil, Ecuador. \\ *Autora para correspondencia; E-mail: mpozo@espol.edu.ec
}

Uno de los valores que tiene el campus Gustavo Galindo, de la Escuela Superior Politécnica del Litoral (Espol), es contar dentro de su predio con un bosque protector de 332,3 ha de bosque seco. En los últimos 5 años se han realizado 10 conteos de aves en los meses de mayo y diciembre, por especialistas y aficionados a las aves. Hasta el momento, se han registrado 163 especies de aves mediante un enfoque cualitativo a través de transeptos lineales de observación. Se incluyen tres especies calificadas como En Peligro de extinción a nivel nacional: Gavilán Dorsigrís Pseudastur occidentalis, Amazona Frentirroja Amazona autumnalis y Cabezón Pizarroso Pachyrhamphus spodiurus; siete especies en categoría Vulnerable: Tinamú Cejiblanco 
Crypturellus transfasciatus, Chachalaca Cabecirrufa Ortalis erythroptera, Perico Caretirrojo Psittacara erythrogenys, Perico Cachetigrís Brotogeris pyrrhoptera, Loro Alibronceado Pionus chalcopterus, Carpintero Guayaquileño Campephilus gayaquilensis y Mosquero Real del Pacífico Onychorhynchus occidentalis. Además, se incluyen 34 especies endémicas del bosque seco tumbesino y una endémica del Chocó. En la actualidad se están diseñando diferentes estrategias de educación ambiental con estudiantes de la Espol y las escuelas cercanas al campus sobre la importancia del bosque seco.

Palabras clave

Aves, bosque seco, Bosque Protector La Prosperina, endemismo, especies amenazadas.

\title{
NUEVOS REGISTROS DE LA ESTRELLITA DE GORGUERA CHAETOCERCUS HELIODOR EN LA CUENCA MEDIA DEL RÍO UPANO, MORONA SANTIAGO
}

\author{
Galo Real Jibaja ${ }^{1}$, Alejandro Solano-Ugalde ${ }^{2}$, Aster Real Wallace ${ }^{1}$ \\ ' RealNature Travel Company, Av. La Ciudad s/n, barrio La Barranca, Macas, Ecuador. \\ ${ }^{2}$ Fundación Imaymana, Paltapamba 476, San Pedro del Valle, Nayón, Ecuador. \\ *Autor para correspondencia; E-mail: galoreal@yahoo.com
}

Existe poca información sobre la Estrellita de Gorguera Chaetocercus heliodor en el sur del Ecuador, por lo que se tenía un conocimiento limitado de sitios para observarla. En esta nota actualizamos el conocimiento sobre su distribución en la provincia de Morona Santiago, donde se había reportado entre 1938 y 1939. Hemos obtenido los siguientes registros en y cerca de Macas: 1) carretera Macas-Riobamba (2009-2013); 2) ciudad de Macas (2010, 2011, 2014); 3) loma del Quilamo (2012-2013); 4) playas del río Upano (2012); 5) Casa Upano (2009-2016). Chaetocercus heliodor ha sido observada con mayor frecuencia en Casa Upano, con más registros en enero-febrero y junio-septiembre. Normalmente, observamos 2-3 individuos. Nuestras observaciones de campo y el registro de avistamientos en eBird por parte de varios observadores de aves y ornitólogos, nos permiten establecer que $C$. heliodor es común y residente alrededor de Macas, con registros en al menos 6 meses del año. Además, nos indica que necesitamos seguir analizando el estado de sus poblaciones en Morona Santiago, y estudiar su historia natural ya que se conoce muy poco sobre su biología reproductiva. Para los observadores de aves que vivimos en Morona Santiago esto es motivante, porque nos permite seguir observando e investigando un poco más de este hermoso colibrí.

Palabras clave

Chaetocercus heliodor, estacionalidad, Estrellita de Gorguera, Morona Santiago, registros.

\section{PARA MUESTRA UN BOTÓN. ¿LA AVIFAUNA DE LAS PISCINAS DE OXIDACIÓN DE SANTA ELENA PODRÍA REFLEJAR LA AVIFAUNA DE LA IBA EC021, VELASCO IBARRA?}

\author{
Enzo M. R. Reyes ${ }^{13^{*}}$, Karina Bazurto ${ }^{2,3}$ \\ ${ }^{1}$ Museo de Ballenas, Av. General Enríquez Gallo 11-09, Salinas, Ecuador. \\ ${ }^{2}$ Reserva Ecológica Manglares Churute, vía a Naranjal, Churute, Ecuador. \\ ${ }^{3}$ Colectivo "Seas and Forests", Santa Elena, Ecuador. \\ *Autor para correspondencia; E-mail: enzorreyesb@gmail.com
}

El presente estudio es parte del programa de monitoreo de aves acuáticas del Museo de Ballenas. Estamos realizando censos mensuales desde 2013 en las piscinas de oxidación de Santa Elena, que constan de cuatro piscinas ubicadas al suroeste de la ciudad, en un área aproximada de 17 ha, cuyo mantenimiento está a cargo de Aguapen-EP (Empresa Pública de Agua Potable de Santa Elena). Empleamos transeptos mensuales de 550 $\mathrm{m}$ por los bordes oeste y norte de las piscinas. Realizamos registros visuales y auditivos de especies acuáticas y de especies terrestres poco comunes. Hasta junio de 2016 hemos registrado 44 especies acuáticas, 45,5\% de ellas migratorias; 29,4\% residentes cuya reproducción en el sitio no se ha registrado; y 13,6\% residentes reproductivas. Nueve especies son consideradas poco comunes en la zona: Tachybaptus dominicus, Anas cyanoptera, Nomonyx dominicus, Fulica ardesiaca, Burhinus surperciliaris, Pluvialis dominica, Calidris 
melanotos, $C$. bairdii y Childonias niger. El número de especies acuáticas reportadas comprende casi la mitad de las especies registradas en un área bastante estudiada como las piscinas de Ecuasal, con poco más de 100 especies. Entre las especies terrestres se incluyen depredadores migratorios como Falco peregrinus y Falco columbarius, y depredadores residentes. Además, encontramos Synallaxis tithys, Sicalis taczanowskii y Phrygilus alaudinus. La finalidad de este estudio es concientizar sobre la importancia del área, protegerla de actividades antropogénicas y discutir su posible incorporación, además de la cuenca del río Salado y el estero de Mar Bravo, dentro del Área de Importancia para la Conservación de las Aves (IBA EC021) Velasco Ibarra, que ha sido pobremente estudiada y que no está protegida. Un censo rápido en Velasco Ibarra en 2013 mostró congregaciones de Fulica ardesiaca, Anas bahamensis, Phalacrocorax brasilianus, Platalea ajaja y Pandion haliaetus en dos cuerpos de agua.

\title{
Palabras clave
}

Aves acuáticas, aves terrestres, conservación, diversidad, piscinas de oxidación de Santa Elena, Represa

Velasco Ibarra, Santa Elena.

\section{ALGUNAS AVES PRESENTES EN EL PARQUE LINEAL SANTA CLARA, SANGOLQUí, PICHINCHA}

\section{María Fernanda Salazar Vaca}

Grupo de Observadores de Aves de Quito.

E-mail: mafer83600@hotmail.com

Uno de los problemas que aquejan a las aves en todo el mundo es la pérdida de bosques nativos o sus remanentes, por diferentes causas. A nivel urbano, la pérdida de hábitat se da principalmente por el creciente desarrollo urbanístico. La comunidad de observadores de aves y muchos aficionados estamos conscientes de esta realidad, por lo cual se han emprendido levantamientos de información de la diversidad de aves en varias localidades urbanas del país. Una de estas iniciativas son los Conteos Navideños de Aves. He realizado conteos navideños de aves, junto con otros observadores aficionados de aves, en un remanente de matorral montano en el Parque Lineal Santa Clara, Sangolquí, provincia de Pichincha. Este parque alberga alrededor de 30 especies de aves. En el presente trabajo se dan a conocer las aves más comunes presentes en estos remanentes de bosque mediante la recopilación de fotografías con información ecológica importante en ciertos casos. Entre algunos de los registros se incluyen especies como Buteo platypterus, Patagioenas fasciata, Falco sparverius, Sayornis nigricans, Diglossa sittoides, Arremon assimilis, Piranga rubra, Pheucticus chrysogaster, Euphonia cyanocephala, entre otras. Esta información se orienta a dar a conocer la importancia del cuidado de las áreas verdes y remanentes de bosque en las ciudades, como refugios clave dentro de las ciudades, evidenciando que la biodiversidad constituye un valor agregado dentro de la zona urbana.

\section{Palabras clave}

Aves, Conteo Navideño, Sangolquí, zona urbana.

\section{ENCUENTROS CERCANOS Y NOTAS SOBRE EL COMPORTAMIENTO DE "SHUNGUITO” Pittasoma rufopileatum: RESERVA MASHPI SHUNGO, PACTO, PICHINCHA}

\author{
Alejandro Solano-Ugalde ${ }^{1,2}$, Danilo Chalá ${ }^{2}$ \\ 'Fundación Imaymana, Paltapamba 476, San Pedro del Valle, Nayón, Ecuador. \\ ${ }^{2}$ Reserva Mashpi Shungo, Mashpi, Ecuador. \\ *Autor para correspondencia; E-mail: jhalezion@gmail.com
}

El presente estudio fue realizado en la reserva Mashpi Shungo, Pacto, provincia de Pichincha. Investigamos la avifauna por cinco años, y hace poco más de un año realizamos el primer registro de un individuo de Pittasoma rufopileatum. Esta especie es endémica de la biorregión del Chocó. En Ecuador es rara, con pocos registros, por lo que mucho de su historia natural se considera un misterio. Actualmente, se considera como 
Casi Amenazada a nivel global y Vulnerable a nivel nacional. Desde mayo de 2015 hasta la fecha hemos mantenido contactos cercanos continuos con un macho adulto, el cual ha sido habituado a recibir presas naturales durante las mañanas. Hasta la fecha se ha tenido un $85 \%$ de éxito en observaciones en campo, documentándose así aspectos previamente desconocidos sobre su historia natural. Se comenta sobre asociaciones con hormigas, mamíferos, actividad nocturna, dieta, percha de dormitorio, vocalizaciones y área de vida.

\section{Palabras clave}

Aves, conservación, Chocó, historia natural, Pittasoma rufopileatum.

\section{AGRADECIMIENTOS}

Los editores agradecen el enorme esfuerzo del comité organizador local de la V REO, en particular a Génesis Romero, Trotsky Riera, Catherine Vits y Paúl Palacios, y al Gobierno Provincial de Zamora Chinchipe, Naturaleza y Cultura Internacional, Copalinga Ecolodge, Universidad Técnica Particular de Loja, Cámara de Turismo de Zamora y Gobierno Municipal de Zamora. Gracias al prefecto provincial de Zamora Chinchipe, Salvador Quishpe, por su compromiso con la V REO. También a las instituciones auspiciantes de la reunión y a todos los participantes. 